\title{
Team AnnieWAY's Autonomous System for the DARPA Urban Challenge 2007
}

\author{
Sören Kammel ${ }^{1}$, Julius Ziegler ${ }^{1}$, Benjamin Pitzer ${ }^{1}$, Moritz Werling ${ }^{2}$, Tobias \\ Gindele $^{3}$, Daniel Jagzent ${ }^{3}$, Joachim Schöder ${ }^{3}$, Michael Thuy ${ }^{4}$, Matthias Goebl ${ }^{5}$, \\ Felix von Hundelshausen ${ }^{6}$, Oliver Pink ${ }^{1}$, Christian Frese ${ }^{3}$, and Christoph Stiller ${ }^{1}$ \\ ${ }^{1}$ Institute for Measurement and Control, University of Karlsruhe, 76131 Karlsruhe, Germany \\ ${ }^{2}$ Institute for Applied Computer Science/Automation, University of Karlsruhe, 76128 Karlsruhe, Germany \\ ${ }^{3}$ Industrial Applications of Informatics and Microsystems, University of Karlsruhe, 76131 Karlsruhe, Germany \\ ${ }^{4}$ Institute for Distributed Measurement Systems, Technical University of Munich, 80290 Munich, Germany \\ ${ }^{5}$ Institute for Institute for Real-Time Computer Systems, Technical University of Munich, 80290 Munich, Germany \\ ${ }^{6}$ Department of Aerospace Engineering, University of the Federal Armed Forces, 85577 Neubiberg, Germany
}

\begin{abstract}
This paper reports on AnnieWAY, an autonomous vehicle that is capable of driving through urban scenarios and that has successfully entered the finals of the DARPA Urban Challenge 2007 competition. After describing the main challenges imposed and the major hardware components, we outline the underlying software structure and focus on selected algorithms. Environmental perception mainly relies on a recent laser scanner which delivers both range and reflectivity measurements. While range measurements are used to provide 3D scene geometry, measuring reflectivity allows for robust lane marker detection. Mission and maneuver planning is conducted via a concurrent hierarchical state machine that generates behavior in accordance with California traffic laws. We conclude with a report of the results achieved during the competition.
\end{abstract}




\section{Introduction}

The capability to concurrently perceive a vehicle's environment, to stabilize its motion and to plan and conduct suitable driving maneuvers is a remarkable competence of human drivers. For the sake of vehicular comfort, efficiency, and safety, research groups all over the world have worked on building autonomous technical systems that can in part replicate such capability (Bertozzi et al., 2000; Franke et al., 2001; Nagel et al., 1995; Thorpe, 1990; Dickmanns et al., 1994).

The DARPA Urban Challenge 2007 has been a competition introduced for expediting research on this kind of systems. Its finals took place on Nov. 3rd, 2007 in Victorville, CA, USA. As in its predecessors, the Grand Challenges of 2004 and 2005 (Darpa, 2005; Thrun et al., 2006), the vehicles had to conduct missions fully autonomously without intervention of human team members (see Fig. 1). In contrast to the earlier competitions, the Urban Challenge required operation in a mock urban scenario, including traffic made up from both competing autonomous vehicles and human driven cars. The major challenge imposed was collision-free driving in traffic in compliance with traffic rules (e.g. right of way at intersections) while completing the given mission. This required for passing parked cars, performing u-turns, parking, and merging into regular flow of traffic. Finally, recovery strategies had to be demonstrated in deadlock situations or in traffic congestions that cannot solely be handled by strictly following traffic rules.

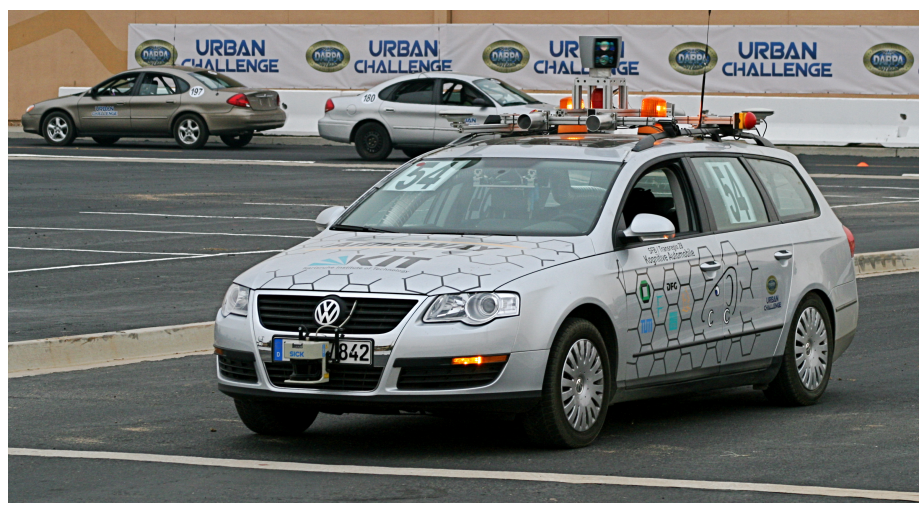

Figure 1: AnnieWAY stopping at an intersection on track A during the NQE.

The scope of Team AnnieWAY was to extract early research results from the Cognitive Automobiles project that would allow real-time operation of the vehicle under the restricted traffic environment in the Urban Challenge. Its team members are professionals in the fields of image processing, 3D perception, knowledge representation, reasoning, real time system design, driver assistance systems and autonomous driving. Some of the team members were in the 'Desert Buckeyes' team of Ohio State University and Universität Karlsruhe (TH) and developed the 3D vision system for the Intelligent Off-road Navigator (ION) that traveled successfully 29 miles through the desert during the Grand Challenge 2005 (Özgüner et al., 2007; Hummel et al., 2006). 


\section{Hardware Architecture}

The basis of the AnnieWAY automobile is a VW Passat Variant (see Fig. 2). The Passat has been selected for its ability to be easily updated for drive-by-wire use by the manufacturer.

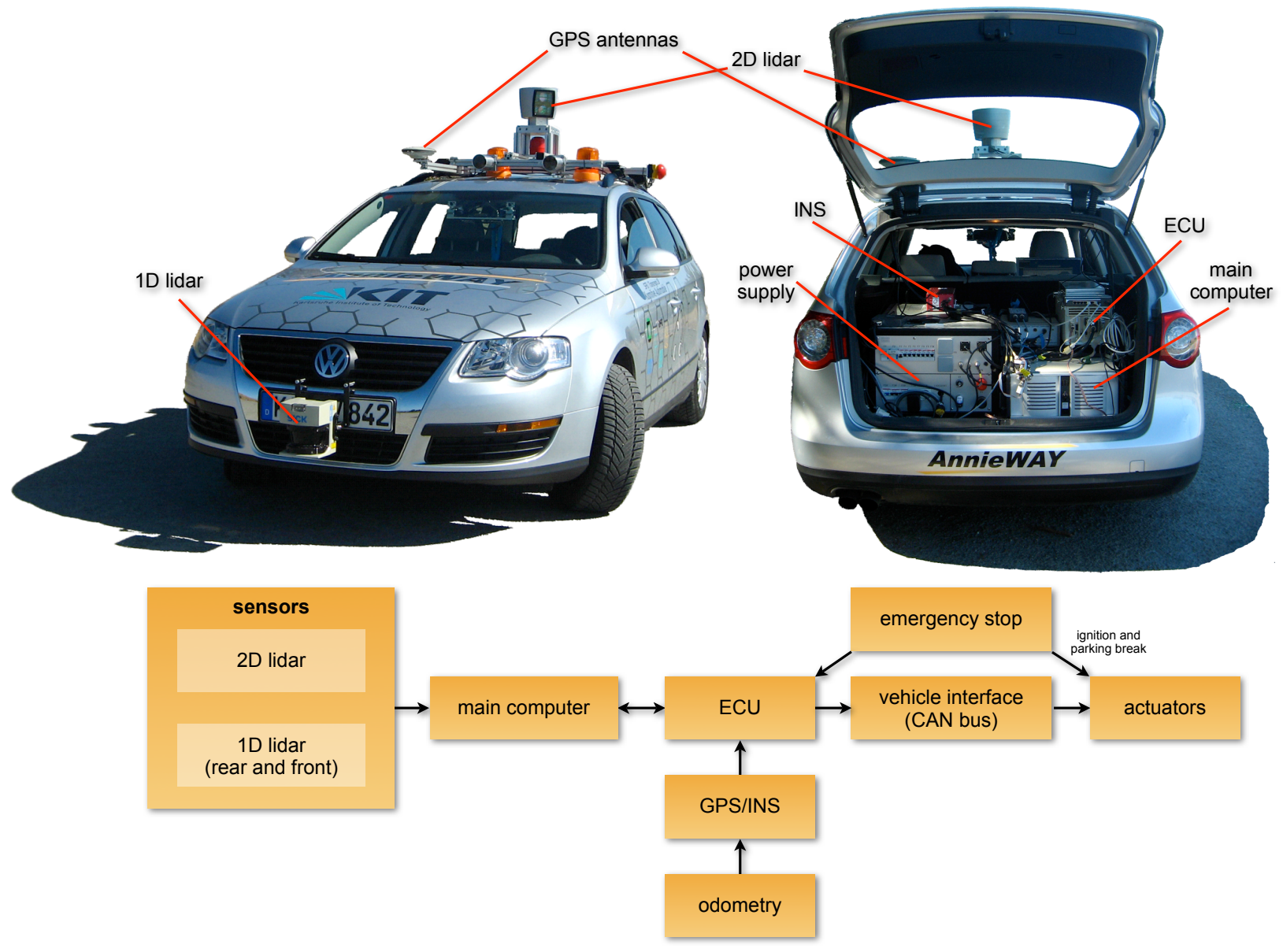

Figure 2: Architecture and hardware components of the vehicle.

\subsection{Computing system}

AnnieWAY relies on an off-the-shelf quad-core computer offering enough processing capacity to run all required software components for perception, situation assessment, and trajectory generation. The chosen hardware architecture is optimally supported by the real-timecapable software architecture which is described in Sec. 3.

The main computer is augmented by an electronic control unit (ECU) for low-level control algorithms. It directly drives the vehicle's actuators. Both computer systems communicate over an Ethernet link. The drive by wire system as well as the car odometry are interfaced via the Controller Area Network (CAN) bus. The DGPS/INS system allows for precise localization and connects to the main computer and to the low-level ECU. 


\section{$2.2 \quad$ Laser-based range and intensity sensors}

Since lidar units produce their own light, low light conditions have no effect on this kind of sensor. In our car we use a rotating laser scanner comprising 64 avalanche photo diodes that are oriented with constant azimuth and increasing elevation covering a $26.5^{\circ}$ vertical field of view. The lasers and diodes are mounted on a spinning platform that rotates at a rate of $600 \mathrm{rpm}$. Thus, the lidar provides a $360^{\circ}$ field of view around the vehicle producing more than 1 million points per second at an angular resolution of $0.09^{\circ}$ horizontally and a distance resolution of $5 \mathrm{~cm}$ with distances up to $100 \mathrm{~m}$. The result is a dense, highly accurate scan representation of almost the entire scene surrounding the vehicle. For each point, the sensor measures range and reflectivity. The reflectivity map is well suited for monoscopic image analysis tasks like lane marker detection. The inherent association of each reflectivity pixel with a range measurement alleviates information fusion of these data significantly. For parking maneuvers, the main lidar is supported by two 2D laser scanners that cover the area directly in front and behind the vehicle.

\subsection{DGPS/INS}

A precise localization is provided by a dead reckoning system which consists of an advanced six-axis inertial navigation system with an integrated RTK/GPS receiver for position and a second GPS Receiver for accurate heading measurements. Odometry is taken directly from AnnieWAY's wheel encoders. The dead reckoning system delivers better than $0.02 \mathrm{~m}$ positioning accuracy under dynamic conditions using differential corrections and $0.1^{\circ}$ heading accuracy using a $2 \mathrm{~m}$ separation between the GPS antennas.

\subsection{Emergency stop system}

As the vehicle had to operate unmanned, a wireless stop system has been integrated for safety reasons as required by the organizer. This E-Stop system allows to remotely command run-, pause-, or emergency-stop mode. The system is connected directly to the ignition and the parking brake to ascertain appropriate emergency stop regardless of the state of the computer system. Run and pause mode are signaled to the low-level control computer. 


\section{Software architecture}

The core components of the vehicle are the perception of the environment, an interpretation of the situation in order to select the appropriate behavior, a path planning component and an interface to the vehicle control. Fig. 3 depicts a block diagram of the information flow in the autonomous system. Spatial information from the sensors is combined to a static 2D map of the environment. Moving objects are treated differently. Such dynamic objects also include traffic participants that are able to move but have zero velocity at the moment. To detect moving objects, the spatial measurements of the lidar sensor are clustered and tracked with a multi-hypothesis approach. To detect possibly moving objects, a simple form of reasoning is used: If an object has the size of a car and is located on a detected lane, it is considered to be probably moving. Lane markers are detected in the reflectance data of the main lidar. Together with the road network definition file (RNDF), the absolute position obtained from the dead reckoning system and the mission data file (MDF), this information serves as input for the situation assessment and the subsequent behavior generation. Most of the time, the behavior will result in a drivable trajectory. If a road is blocked or the car has to be parked, modules for special maneuvers, like the parking zone navigation module, are activated.

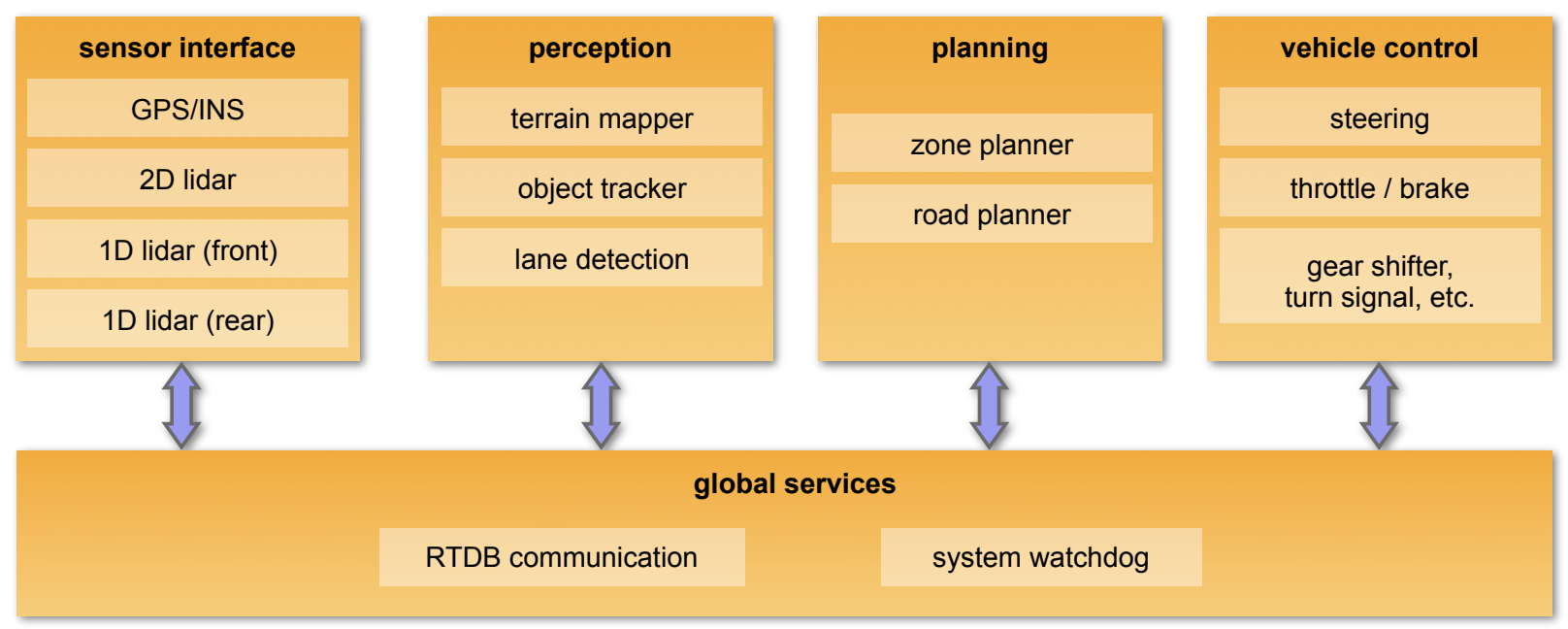

Figure 3: Overview of the software architecture and the information flow.

All data exchange between processes is done via a central communication framework, the real-time database for cognitive automobiles KogMo-RTDB (Goebl and Färber, 2007b). All data within the RTDB is represented as time-stamped objects. The centralized data storage gives the opportunity to easily log and replay all or selected objects. For performance reasons the database is completely memory based. It is capable of distributing even large data objects, like lidar raw sensor data, to several processes and at the same time relay vehicle control commands at a rate of $1 \mathrm{kHz}$ between a vehicle control process and the ECU (Goebl and Färber, 2007a). 


\section{Perception}

\subsection{Environmental mapping}

Accurate and robust detection of obstacles at a sufficient range is an essential prerequisite to avoid obstacles on the road and in unstructured environments like parking lots. The basic idea is to maintain an evenly spaced 2D grid structure $g$ where each cell $g_{i}$ represents a random variable. Each random variable is binary and corresponds to the occupancy it covers. Therefore, in the literature this approach is also called occupancy grid mapping ((Thrun, 2002; Thrun, 2003)) which has the goal to calculate the posterior over maps $p(g \mid \mathbf{z}, \mathbf{x})$ where $\mathbf{z}$ is the set of all measurements and $\mathbf{x}$ is the path of the vehicle defined through a sequence of poses. An example of a resulting evidence map is depicted in Fig. 4.

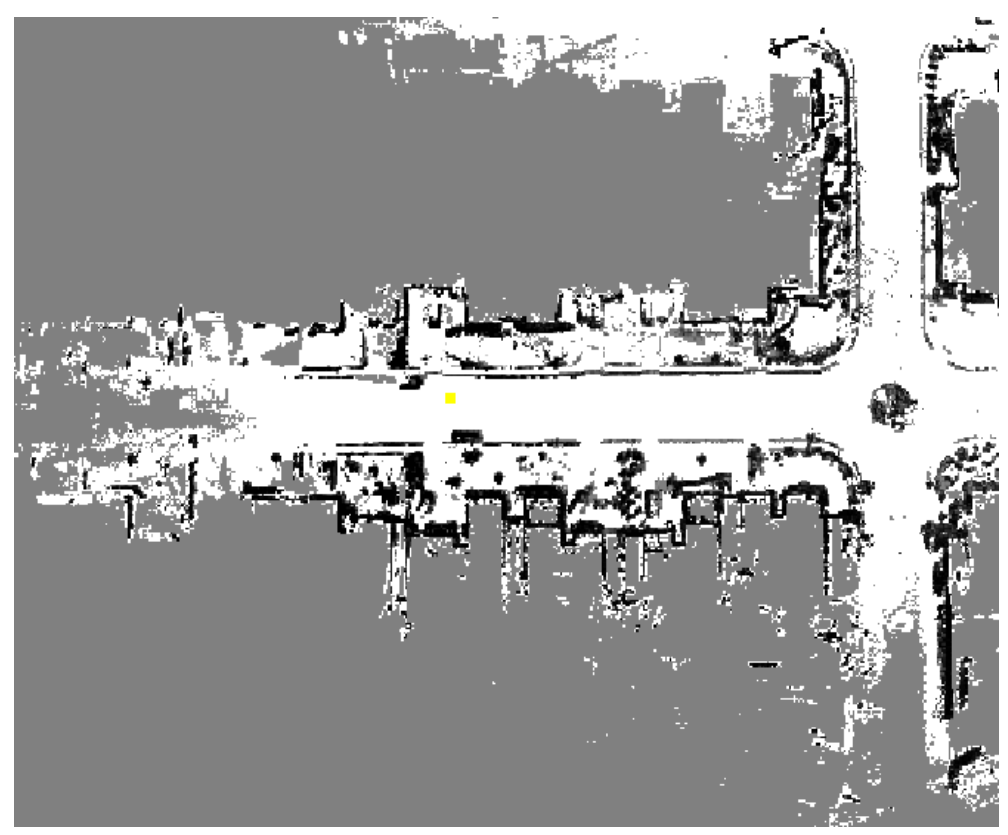

Figure 4: Example for the evidence mapping of 3D lidar data onto a 2D grid. Darker spots correspond to high evidence for an obstacle while white cells correspond to drivable area. Unknown cells are marked as grey.

AnnieWAY uses a grid that is always centered at the vehicle position but aligned with a global coordinate system. The grid is shifted at each time step to account for the new vehicle position. This restricts the size of the map to an area around the vehicle while the cells are bound to an absolute position. The size of each grid cell is $15 \mathrm{~cm} \times 15 \mathrm{~cm}$. Fig. 5 shows an example of our mapping algorithm. The grid is generated mainly from multi-layer, high resolution lidar data. Algorithms for the integration of low resolution lidar data can be found in (Thrun, 2002; Thrun, 2003; Biber and Strasser, 2006; Bosse et al., 2003).

Integrating the data of the laser scanners into an environmental map consists of three steps. In the first step the range measurements $z_{l \in L}$ of one revolution $L$ are projected into a global coordinate system under consideration of the vehicle's motion $x_{l}$. In the second step, different measures are extracted from the data for each cell $g_{i}$. Two straightforward measures are the 
number of measurements $n_{i}$ and the number of different laser beams $b_{i}$. The most important measure we use is the elevation difference

$$
e_{i}\left(g_{i}, z_{l}\right)=\max _{l \in L} h\left(g_{i}, z_{l}\right)-\min _{l \in L} h\left(g_{i}, z_{l}\right)
$$

where $h$ is the vertical component of each measurement.

In the third step, we compute the evidence for each measure by using an inverse sensor model. E.g. the inverse sensor model for the elevation difference returns $l_{\text {occ }}$ if $e_{i}$ exceeds a certain threshold (e.g. $15 \mathrm{~cm})$ and $l_{\text {free }}$ otherwise. The inverse models for $n_{i}$ and $b_{i}$ are slightly more complex since they are learned by a supervised learning algorithm. The result of the learning procedure is a forward model that accepts $g_{i}$ and $n_{i}$ or $b_{i}$ respectively as parameters and returns the appropriate evidence.

Finally, we can compute the combined occupancy evidence $o_{i, t}$ as a weighted sum of the three partial evidences:

$$
o_{i, t}=o_{i, t-1}+\alpha_{1} \cdot n_{i}+\alpha_{2} \cdot b_{i}+\alpha_{3} \cdot e_{i}
$$

and the estimated occupancy for a single cell

$$
p\left(g_{i} \mid \mathbf{z}, \mathbf{x}\right)=1-\frac{1}{1+\exp o_{i}}
$$

As already mentioned, AnnieWAY is equipped with different sensors and ideally one wants to integrate information from all sensors into a single map. A naive solution is to update the map for each sensor separately which neglects the different characteristics of each sensor, e.g. field of view, maximal range and noise characteristic. To ensure safe driving we use the most pessimistic approach to fuse sensor data: We compute the maximum of all estimated occupancies, where $K$ is the number of sensors:

$$
p\left(g_{i}\right)=\max _{k \in K} p\left(g_{i}^{k}\right)
$$

If any sensor detects a cell as occupied it will be occupied in the combined map.

The standard occupancy grid mapping algorithm suffers from a major drawback: it is only suitable for static environments. Driving environments are typically highly dynamic and the result is very poor without modifications. Moving objects create virtual obstacles with high evidence while moving. To overcome this problem we introduce a temporal evidence decay. The evidence is reduced at each time step by a factor $\epsilon_{t}$ for cells which are not updated. The intuition is that the uncertainty increases for cells not augmented by any sensor. Equation 2 turns now into 


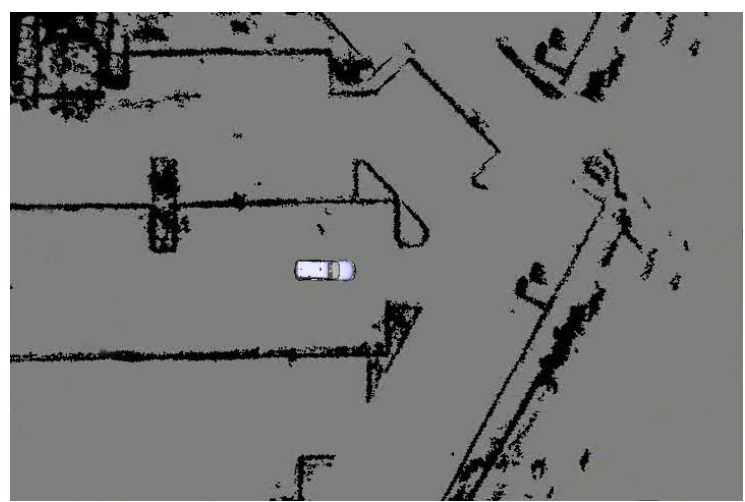

(a) Map generated from a parking lot.

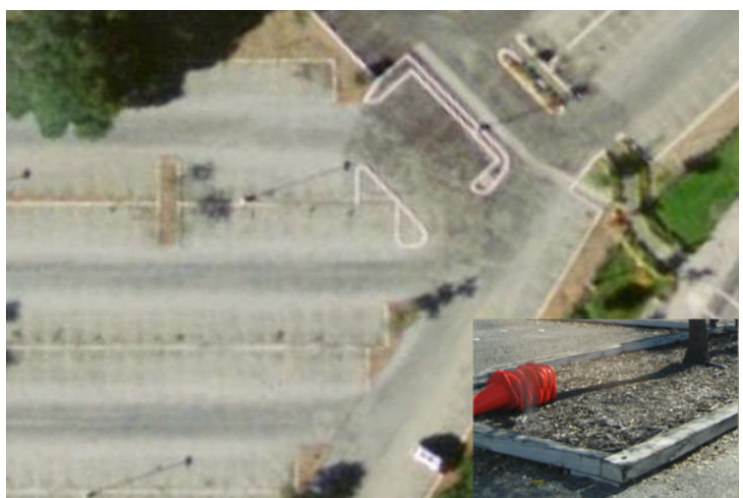

(b) Aerial imagery of the parking lot with a detail photo of the curb in the lower right corner.

Figure 5: Example for a generated evidence map and an aerial image of the corresponding region.

$$
o_{i, t}=\operatorname{argmax}\left(0, o_{i, t-1}+\alpha_{1} \cdot n_{i}+\alpha_{2} \cdot b_{i}+\alpha_{3} \cdot e_{i}-\epsilon_{t}\right),
$$

where the argmax operator enforces positive evidences.

\section{$5 \quad$ Tracking of dynamic objects}

Driving in urban environments requires to capture and estimate the dynamics of other traffic participants in real-time. AnnieWAY uses a processing pipeline that takes in raw sensor data (from different lasers) and generates a list of dynamic obstacles, along with their estimated locations, sizes, and relative velocities. This pipeline consists of a number of parts, including

1. Data preprocessing: removing irrelevant readings: noise, ground readings, readings from obstacles outside the road, etc.

2. Obstacle detection: creating a list of obstacles raw readings...includes segmentation for laser

3. Obstacle tracking: corresponding obstacles time step with those of another time step in order determine their headings, relative velocities, etc.

\section{Obstacle post-processing and publishing}

The data preprocessing step used for tracking was discussed earlier as part of Sec. 4.1. The result of this part is a grid map with occupancy probabilities attached to each cell. All the sensors' information has been condensed within this grid.

The first stage of dynamic object tracking is the object detection which is - in the sense of a statistical approach - equivalent to the identification of object hypothesis. AnnieWAY uses an occupancy grid map which has been segmented using a connected components approach. Therefore, we treat each grid cell as a node in a graph $G$. Two points are connected if and 


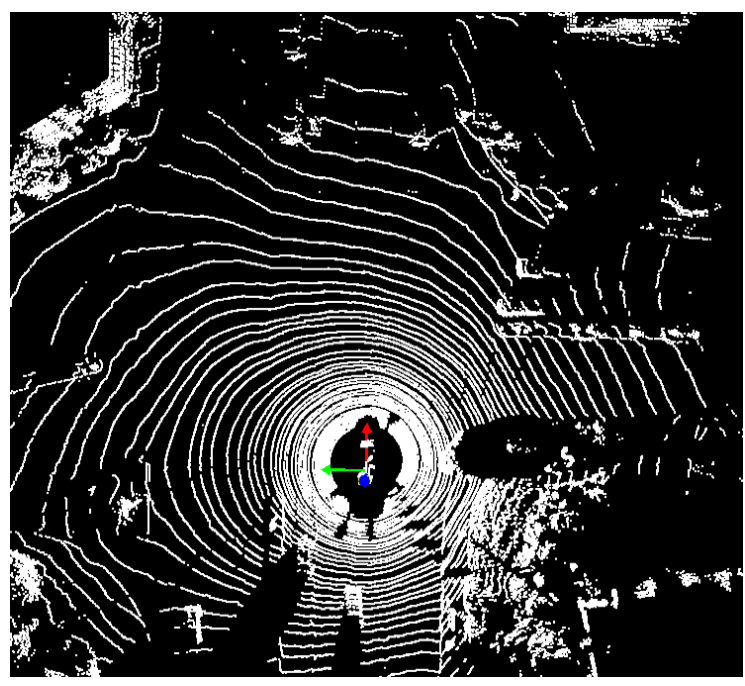

(a) High resolution lidar data.

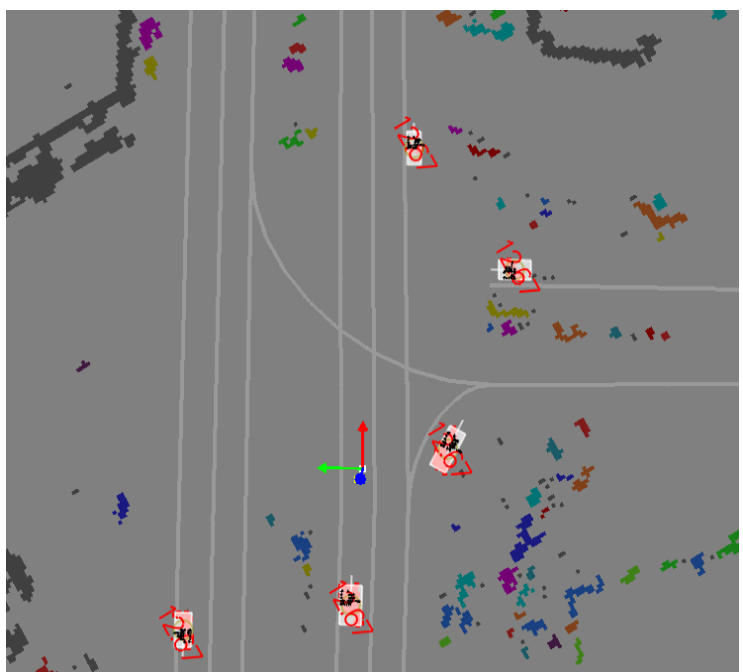

(b) Tracked vehicles on segmented grid map.

Figure 6: Tracking of dynamic objects with occupancy grid map and linear Kalman Filter.

only if the distance between them is within a threshold $d$ (e.g. $0.5 \mathrm{~m})$. We then find all the connected components in the graph and assign the same label to those cells. To reduce noise, we discard any connected component with less than a minimum number of cells. Due to the uniform angle resolution of the scanners, the number of cells an object consists of depends on its distance. The closer an object is located to the scanner, the more laser rays will hit the object.

The connected components are analyzed in a second step for their probability of being a traffic participants. Several heuristics are used based on their shape and location relative to the road network. Only 'good' candidates are augmented in the following tracking step. Fig. 6(b) displays the resulting objects after post-processing.

With this procedure, not all captured and tracked objects are relevant to be published to other modules. This is due to noisy observations, occlusion, dynamic objects eaving of our sensors' fields of view, etc. All these effects lead to unlikely object hypotheses, but nevertheless they are internally tracked. In order to decide when to publish relevant obstacles, we define a notion of confidence that works similarly to log-likelihood updates in an occupancy grid map as mentioned earlier. If an obstacle is observed, we increment its confidence, in case it goes unobserved in our field of view, we decrement it. Thus defined, the confidence allows us to set minimum thresholds for the tracking and publishing obstacles: if the object's confidence exceeds the threshold, the obstacle is published to all other attached modules. If its confidence undercuts a certain threshold, the object is removed from the obstacle list. Hypotheses within the both thresholds are internally tracked, but not published.

Tracking of dynamic objects mainly serves two purposes. First, it aids the correspondence of obstacles detected in one sensor frame at time $t=k$ with those in subsequent sensor frames at time $t=k+1$. This can be easily achieved with distance-based methods or more sophisticated 3D fitting and registration algorithms like iterative closest point (ICP). 
However, these methods do not take into account the noise and uncertainty of our sensors. The second and equally important purpose of tracking is to return estimates of other vehicle's relative velocities and headings.

AnnieWAY uses a linear Kalman filter (Kalman, 1960) to model a simplified dynamic obstacle with its appropriate state vector $\left[\begin{array}{llll}x, & y & \dot{x} & \dot{y}\end{array}\right]^{T}$. Obviously, this model ignores completely the underlaying physical and non-linear behavior of a car, but the frequency of sensor updates $(10 \mathrm{~Hz})$ means that cars move very little between them which allows us to assume linear dynamics. Transition updates are linear with an overlaid Gaussian noise characterized by its covariance matrix $\mathbf{Q}$ :

$$
\mathbf{T}=\left[\begin{array}{cccc}
1 & 0 & \Delta t & 0 \\
0 & 1 & 0 & \Delta t \\
0 & 0 & 1 & 0 \\
0 & 0 & 0 & 1
\end{array}\right], \mathbf{Q}=\left[\begin{array}{cccc}
0 & 0 & 0 & 0 \\
0 & 0 & 0 & 0 \\
0 & 0 & \sigma_{q, \dot{x}}^{2} & 0 \\
0 & 0 & 0 & \sigma_{q, \dot{y}}^{2}
\end{array}\right]
$$

Since we are extracting the obstacle's position $\left[\begin{array}{ll}x, & y\end{array}\right]^{T}$ from the measurement, the observation matrix $\mathbf{O}$ looks as described below. Further, we assume mutual independent Gaussian noise sources characterized by the covariance matrix $\mathbf{R}$ :

$$
\mathbf{O}=\left[\begin{array}{llll}
1 & 0 & 0 & 0 \\
0 & 1 & 0 & 0 \\
0 & 0 & 1 & 0
\end{array}\right], \mathbf{R}=\left[\begin{array}{cc}
\sigma_{r, x}^{2} & 0 \\
0 & \sigma_{r, y}^{2}
\end{array}\right]
$$

After performing an observation, we do not know which detected obstacles within the measurements are already tracked or if they are new objects. Thus, we are required to solve a problem of correspondence between observations and the internally tracked dynamic obstacles. This is a nontrivial problem, requiring that we define both a measure of distance and a procedure for finding the optimal correspondence. AnnieWAY uses a maximum-likelihood matching algorithm to find the optimal assignment of observations to existing Kalman filters. This matching is a one-to-one function from filters to observations.

\section{$6 \quad$ Lane marker detection}

Digital maps of a road network are often not up-to-date or resemble the real road network only approximately. Therefore, a local offset between the digital and the real road network may exist. The detection of lane markers helps to minimize this offset. An accurate and continuous detection of lane markers even enables the creation of new road network maps.

In the context of this paper, lane markers can be either painted markings or curbs. Painted lane markings are detected within the intensity readings of the lidar whereas curbs cause small height changes in the range data of the lidar. A combined intensity/range plot is depicted on the left side of Fig. 8. Both kind of lane markers form one dimensional structures that can be approximated by line segments locally. In contrast to camera based intensity 
images, the laser reflectivity and range data is insensitive to background light and shadows. However, the sensor samples the road very sparsely, especially at distance. In order to increase the density of lane marker information, subsequent scans are registered spatially and accumulated employing absolute positioning information from the dead reckoning system. The first step in order to obtain a dense bird eye's view representation of lane marker features is a classification of data points in each scan into obstacle and ground by the algorithms described in Sec. 4.1. Lane markers are expected to occur on the road surface (painted markers) or at its borders (curbs) only. Therefore, points of each individual laser labeled as ground are searched for large continuous chunks (chunks that do not exhibit height changes exceeding the height of curbs) representing the road. Only within those large chunks high intensity gradients are detected. In addition, only measurements exhibiting absolute intensities larger than the median intensity of each laser scan are taken into account. Both types of features - painted markings and curbs - are mapped into a feature grid $g(\mathbf{x})$ similar to the evidence grid described in Sec. 4.1, see Fig. 8(right). Features are detected first in the single scans and mapped afterwards (instead of creating a dense map first and extracting the features afterwards) to minimize the effect of errors in the vehicle localization. A summary of the detection algorithm is shown in Fig. 7.

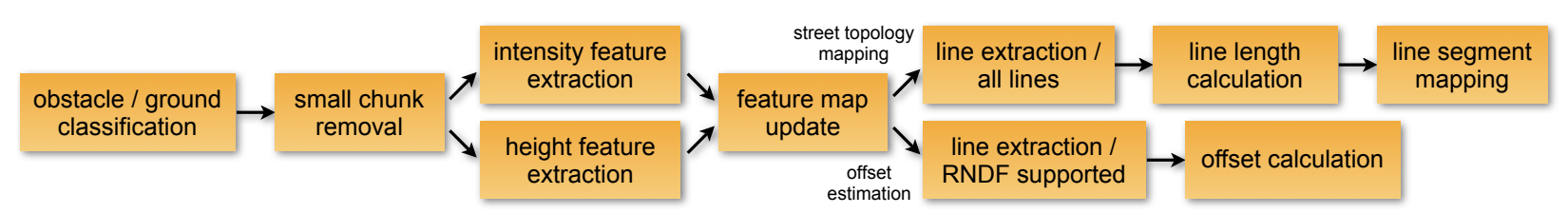

Figure 7: Overview of the offset estimation and street topology mapping

Lane segments are detected by applying the Radon transform to the accumulated feature map data. Since the Radon transform is an algorithm operating globally on the map it proved to be robust against occlusions, noise and outliers. Compared to the Hough transform, the Radon transforms exhibits the advantage of a calculation time independent of the numbers of lane markers and the capability to handle gray-scale images efficiently and without thresholding. For a real-time calculation in the car, an implementation exploiting the central-slice theorem was used (Bracewell, 1990). The position and direction of lane boundaries can be calculated by locating their corresponding maxima in the Radon plane. Since we observed a systematic error of RNDF data in some areas, it appeared sensible to determine a correcting offset from the detected lane markings. To accomplish this, the lane markers specified in the RNDF are first projected into the Radon plane. Assuming that the offset of the road map data does not exceed one lane width, the deviation is obtained in a second step from the distances to the maxima in the Radon plane closest to the predicted positions. Assuming further that predicted and estimated lane boundaries are close to parallel, the vertical distance is sufficient to determine the offset. 

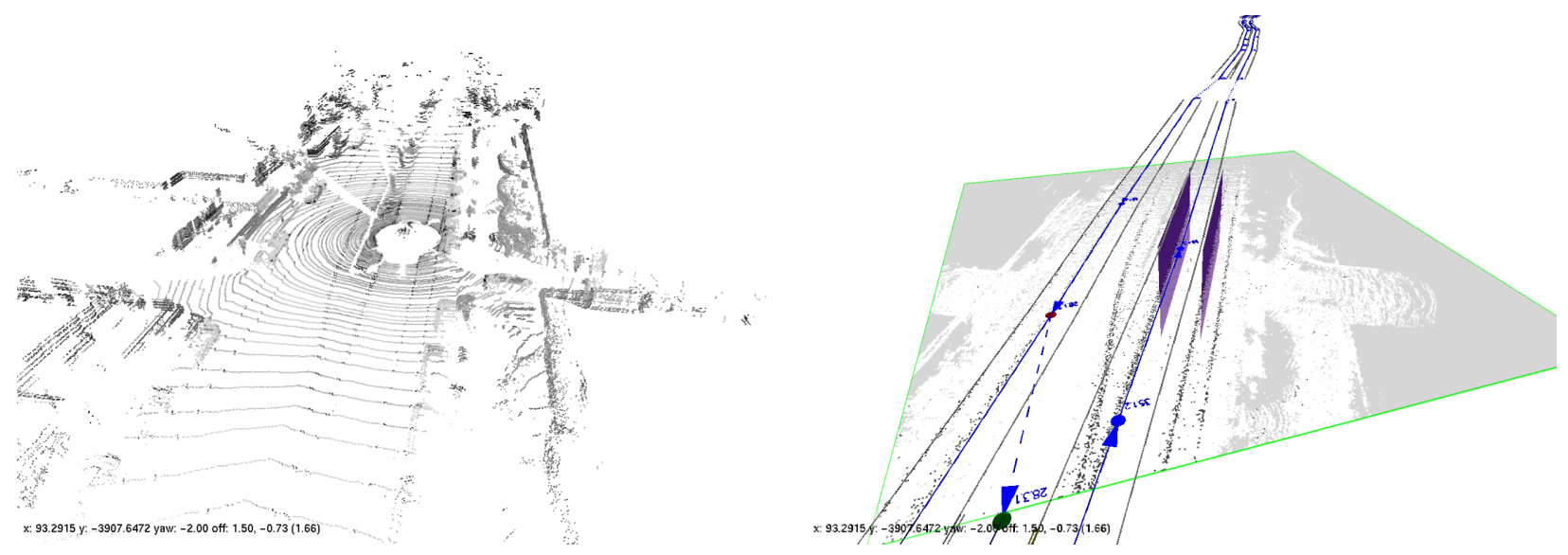

Figure 8: Combined range and intensity readings of the lidar (left) and lane marker map with the estimated current lane segment and an overlay of a part of the original road network map (right). 


\section{$7 \quad$ Reactive Layer}

Our system integrates a reactive layer that allows AnnieWAY to modify a planned trajectory based on GPS waypoints. While the obstacle tracker easily handles objects like cars, small or extended objects like rocks or pavement edges are more difficult to track explicitly. Hence, we integrated a reactive mechanism that gets as input a vehicle-centered occupancy grid (built from the lidar data) and the trajectory planned so far. The algorithm then first evaluates, whether the given trajectory is clear and - only if not - starts a more complex evaluation of the grid that results in a modification of the initially given trajectory. This mechanism is biologically motivated and resembles an insect's use of its antennae to avoid obstacles. What are antennae in nature, are precomputed trajectory primitives (we call them tentacles) in our system. Here, all tentacles are simple circular arcs, but depending on the speed of the vehicle, the parameters of these arcs vary such that at high speeds no dangerous actions can be taken (see Fig. 9). To select the appropriate primitive the occupancy grid is investigated in an area around and underneath that primitive. The final selection is done on the basis of four aspects:

1. Could the vehicle drive the primitive without causing damage? In particular, within a distance the vehicles needs to stop, is the ground along the tentacle clear of anything having a height above $0.1 \mathrm{~m}$ ?

2. How smooth is the terrain under the primitive?

3. How far is the next obstacle along that primitive?

4. How well does the primitive follow the original trajectory?

By considering these aspects as detailed more precisely in (v. Hundelshausen et al., 2008) the vehicle follows the given trajectory if possible, but avoids obstacles, if not. To coordinate this reactive layer with the obstacle tracker, tentacles were only evaluated up to the first explicitly tracked obstacle. In this way, only unexpected obstacles were avoided.

As detailed in (v. Hundelshausen et al., 2008) the overall reactive mechanism was tested excessively by intentionally defining bad GPS-trajectories, e.g having a large offset to the real road (passing through the front gardens of neighboring houses), passing through a traffic circle (instead of leading around it), abbreviating a crossing through a complete house, and other tests including moving vehicles. At the final of the urban challenge this mechanism was important at narrow passages.
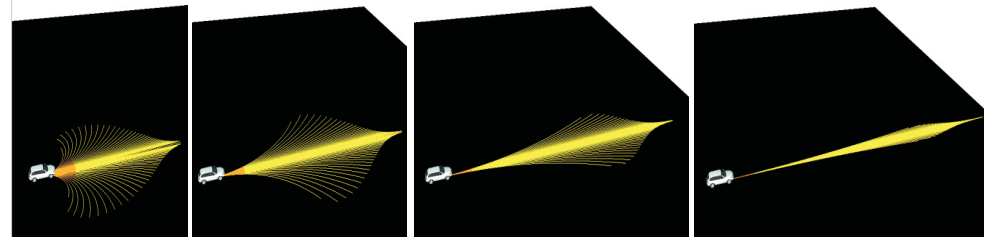

Figure 9: The reactive system uses a precomputed set of motion primitives that vary with the speed of the vehicle. As detailed in (v. Hundelshausen et al., 2008), those primitives are used to evaluate a vehicle-centered occupancy grid to avoid obstacles. 


\section{Planning}

The major challenge imposed by the competition was collision-free driving in traffic in compliance with traffic rules, e.g. right of way at intersections. It included special maneuvers, like overtaking, u-turns, parking, and merging into regular flow of traffic while completing the given missions. To accomplish this, the robot must be capable of analyzing the situation, assessing developments, choosing the appropriate behavior and executing it in a controlled way. AnnieWAY uses a planning module organized in three layers to address these problems:

1. Mission Planning computes a strategic plan to accomplish mission

2. Maneuver Planning applies California traffic rules and plans actual driving maneuvers (e.g. turns, intersection, passing) and generates a corresponing path.

3. Collision Avoidance tests whether the planned path is collision free taking into account the obstacle map acquired from the perception module. If a collision is probable it chooses an alternative path.

In a first preprocessing step, all elements of the RNDF (lanes, checkpoints, exits, etc.) are converted to a graph-based, geometrical representation. RNDF waypoints form the vertices of the graph; lanes and exits are represented by graph edges. In addition to waypoints, a number of vertices interpolating between waypoints are automatically added to the graph. We make use of the geometric structure of roads to insert these supporting waypoints with a polynomial road model (splines). In addition to this, we add virtual turnoff lanes at intersections. A virtual lane connects each exit waypoint with a corresponding entry waypoint. It is generated assuming standard intersection geometry. Information such as distances, lane boundaries, and speed limits annotate the respective graph edges. These annotations can be updated dynamically, to incorporate results from the perception module (e.g. road blockages).

Dynamic objects recognized by the perception module are matched to the most probable edge of the geometrical graph representation, based on their position and orientation. This allows for attributing a role to every object, e.g. identification of a leading vehicle, or semantically localizing an object within an intersection scenario.

Mission Planning is the most abstract form of planning used by AnnieWAY. It finds the optimal route from one checkpoint to another using an $\mathrm{A}^{*}$ graph search algorithm operating on the internal representation of the road network. The heuristic for the search is the minimal travel time to the goal based on the Euclidean distance whereas the costs are defined by the estimated travel time for all edges traversed. The search process is repeated for every pair of subsequent checkpoints in the MDF. In this way the mission planner finds the optimal route traversing all mission checkpoints. Generally the mission planner runs only once while loading the mission file and whenever AnnieWAY has to diverge from the planned route caused by situation dependent reasons (e.g. road blockades). The route is passed on to the downstream maneuver planning as a sorted list of edges to be traversed.

The high-level plan and the AnnieWAY's current position is used by Maneuver Planning to compute actual driving maneuvers. The maneuver planner is implemented as a Concurrent 
Hierarchical State Machine (CHSM) with every state representing a driving behavior. The key aspect of a hierarchical state machine is to design and group the states in a way that a sub-state is a specialization of its parent state, and only extensions to the more general behavior of the parent state have to be modeled explicitly. Thereby, the functional redundancy of the states and the amount of transitions is reduced, so it is easier to capture the complex reactional behavior of a system. Fig. 10 shows the UML state chart of the machine's main level, with important sub-states annotated as well.

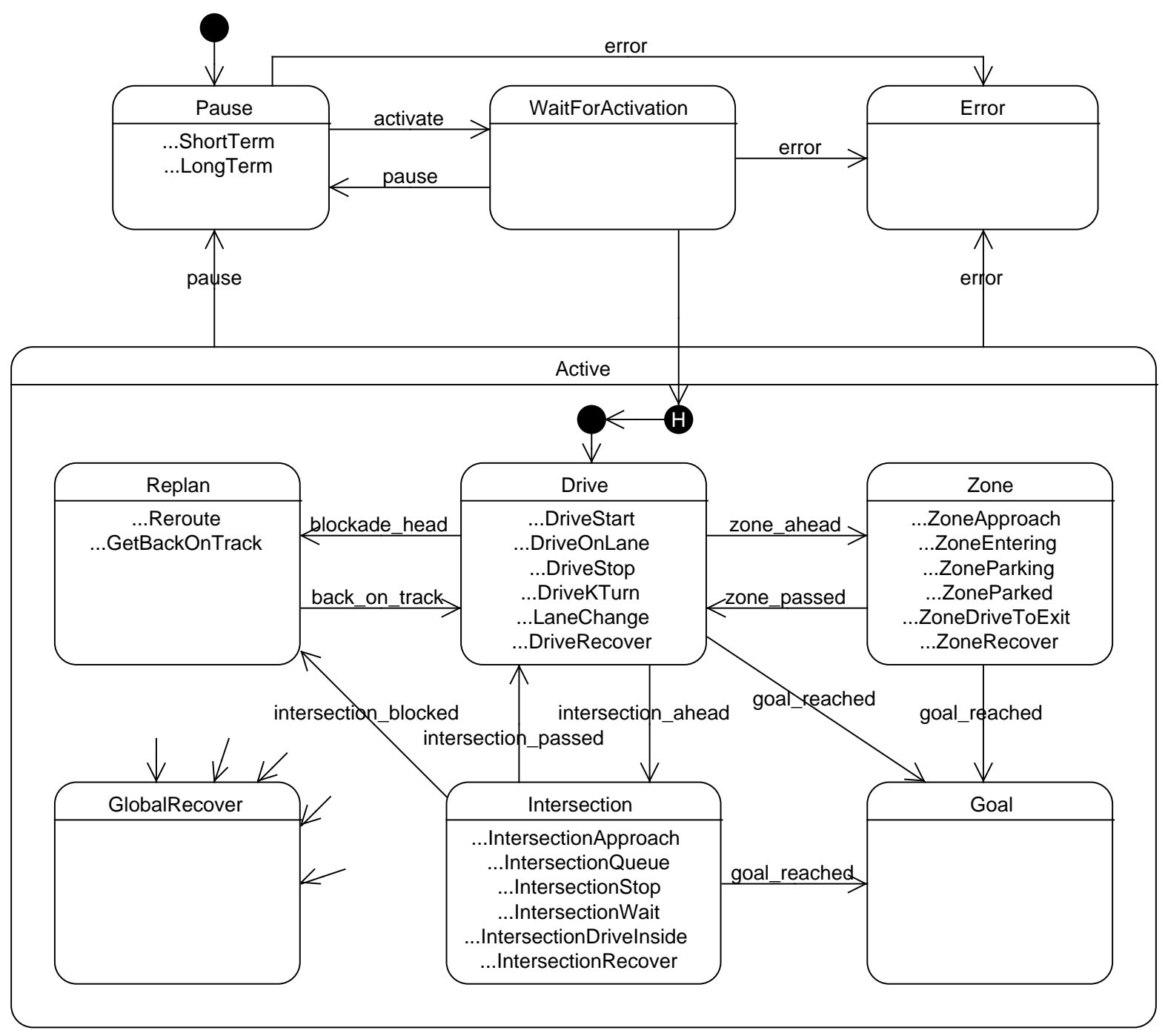

Figure 10: Overview of the concurrent hierarchical state machine used to model traffic situations and behavior

Every behavior the car is capable of, is modeled as a state organized within a state hierarchy. The state Drive comprises all regular driving maneuvers on normal roads. It has several sub-states that cover different situations like following the course of a lane (DriveOnLane), making a k-turn (DriveKTurn) or changing the lane (LaneChange). All behavior at intersections is handled by the Intersection state. It comprises some specialized sub-states for 
different types of intersection. Some more insight on the real functionality and architecture of the state machine is given in Sec. 9, where handling of moving traffic in an intersection scenario is explained in detail. The navigation in unstructured environments and parking maneuvers is controlled by the state Zone and its sub-states. These states control invocation of the navigation module described in Sec. 10. In some situation it becomes necessary for the robot to replan its route, e.g when the road ahead is blocked. This is triggered by the state Replan, that re-activates the mission planning module. Most states implement a recovery state that is activated whenever the car makes no progress at all for a certain amount of time. If all situation dependent recovery handling fails, a global recovery state is invoked to navigate back on track using the navigation module.

When all situation assessment has taken place and all state transitions are made, the reached state generates a path stub, that is input to the closed loop control module (Sec. 11). It reaches approximately $30 \mathrm{~m}$ ahead and consists of densely sampled waypoints combined with heading and curvature information. In the most common case, when the car is driving on roads stored within the graph representation, the trajectory is generated in a straight forward way by sampling the graph edges ahead. These points are smoothed by a spline approximation to generate a continuous curvature path. In areas that lack road geometry description and whenever sensible localization within the road network graph is not possible, the free navigation module in Sec. 10 is used to plan a collision free path to a given target configuration.

Paths generated by the state machine may be overwritten by the low level avoidance system described in Sec. 7. 


\section{Moving traffic}

This section describes an algorithm which reduces dynamic maneuvers, such as merging into moving traffic and crossing intersections with oncoming traffic, to static maneuvers, such as simple turns. Unfortunately, the actual behavior of the other traffic participants cannot be exactly predicted. Therefore certain assumptions, simplifications, and conservative estimates have to be made in an appropriate way, such that the unmanned vehicle operates safely as well as effectively.

\subsection{Problem abstraction and simplifications}

In the following, it is assumed that (1) the other traffic participants with the right of way neither slow down nor speed up, (2) stay in the middle of the road, (3) AnnieWAY's longitudinal controller accelerates at a known constant rate until the desired maneuver velocity is met, and (4) all traffic participants' velocities and positions are known.

Assumption (1) and (2) have to be made, since the actual behavior of the other vehicles $\left(B_{i}\right)$ cannot be precisely predicted. Therefore it is assumed, that the considered vehicles travel at a constant velocity in the center of the priority road. Introducing $t_{\overline{B P}}$ as the time needed for traveling a distance $d_{\overline{B P}}$ in the road center and $v_{B}$ as the other vehicle's constant velocity, leads to

$$
t_{\overline{B P}}=\frac{d_{\overline{B P}}}{v_{B}}
$$

Assumption (3) is based on the longitudinal control strategy, which is described in Sec. 11. The resulting drive-off characteristic $v(t)$ from a start velocity $v_{0}$ to a new desired velocity $v_{d}$ can be seen on the left in Fig. 11 as a dashed line along with the approximation $\hat{v}(t)$ as a solid line.
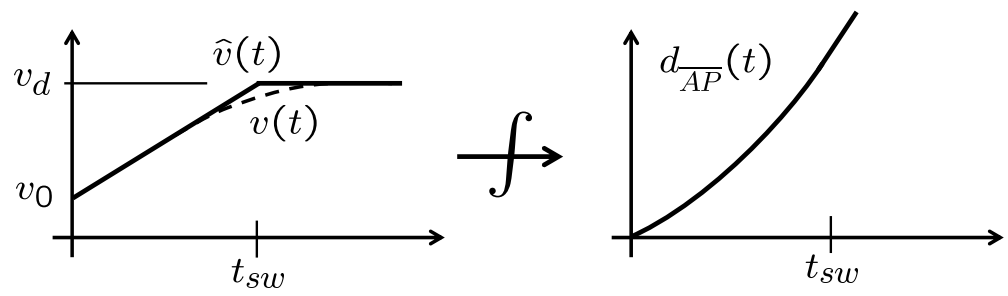

Figure 11: Actual and approximated drive-off characteristic

Here $t_{s w}$ denotes the time, when the approximated velocity $\hat{v}(t)$ reaches $v_{d}$. It can be calculated by

$$
t_{s w}=\frac{v_{d}-v_{0}}{a_{\mathrm{sat}}} .
$$

An integration of $\hat{v}(t)$ over time (see Fig. 11) yields the traveled distance of AnnieWAY (A)

$$
d_{\overline{A P}}(t)=\left\{\begin{array}{cc}
v_{0} t+\frac{a_{s a t}}{2} t^{2} & t \leq t_{s w} \\
v_{0} t_{s w}+\frac{a_{s a t}}{2} t_{s w}^{2}+v_{d}\left(t-t_{s w}\right) & t>t_{s w}
\end{array}\right.
$$




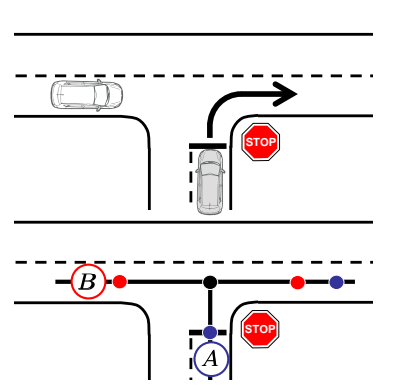

(a) Right turn with stopping

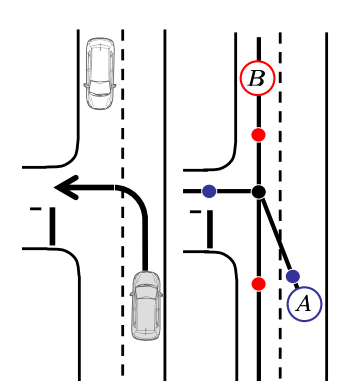

ping

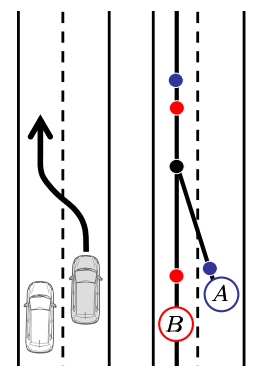

(c) Lane change maneuver

(d) Double lane change maneuver with oncoming traffic.

Figure 12: Different moving traffic scenarios

Solving (10) for $t$ with

$$
t_{a}=\frac{d_{\overline{A P}}-v_{0} t_{s w}-\frac{a_{s a t}}{2} t_{s w}^{2}}{v_{0}+a_{s a t} t_{s w}}+t_{s w}
$$

yields

$$
t_{\overline{A P}}=\left\{\begin{array}{cl}
t_{a} & t_{a}>t_{s w} \\
\frac{1}{a_{s a t}}\left(-v_{0}+\sqrt{v_{0}^{2}+2 a_{s a t} d_{\overline{A P}}}\right) & t_{a} \leq t_{s w}
\end{array}\right.
$$

whereas the ambiguity of the solution was resolved.

Fig. 12 illustrates the transfer of different traffic scenarios to the equivalent graphs, whose generic graph can be found left in Fig. 13 along with the four relevant quantities to be measured, the current distances $d_{A}(t)$ and $d_{B}(t)$ to $M P$, and the current velocities $v_{A}(t)$ and $v_{B}(t)$ (assumption (4)). As can be seen, traffic participants are all assumed to be point masses. Based on the previous equations and graphs, the movement of the vehicles can be predicted and used for collision detection in the next section.

\subsection{Spatial and temporal verification}

On the one hand, at low speed it has to be guaranteed that the autonomous vehicle avoids collisions by not getting too close to other traffic participants. Therefore spatial safety distances were introduced (see Fig. 13, right-hand side). On the other hand, spatial safety distances are not a proper measure at higher speeds. In this case a temporal safety distance assures certain time gaps between AnnieWAY and the other traffic participants. Since time gaps become too small referred to the ground at low speed in turn, both spatial and temporal conditions have to be fulfilled at the same time.

For simplicity's sake only a single vehicle is considered initially. In order to be the first to enter the critical area, the following two conditions have to be met:

1. At time $t_{\overline{B P}}$, when $\mathrm{B}$ reaches $P_{B 1}$, A has to be beyond $P_{A 2}$.

$$
\text { free }_{\mathrm{spat}, A B}=\left(d_{\overline{A P}}\left(t_{\overline{B P}_{B 1}}\right)>d_{A}+D_{A 2}\right)
$$




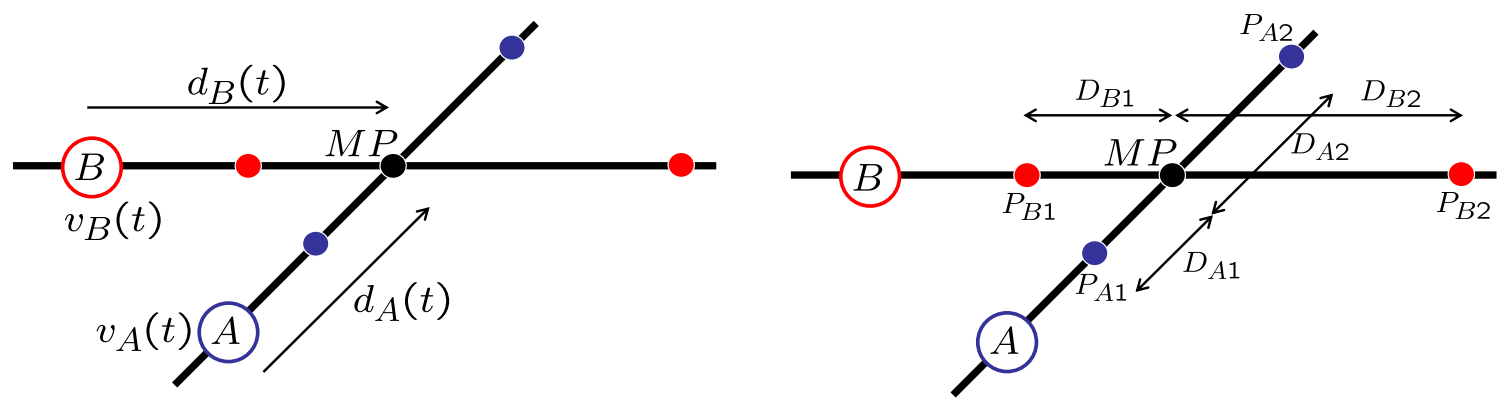

Figure 13: Measured quantities and geometric parameters of the graph

2. After A has passed $M P$, a given time span $\Delta T_{A B}$ has to elapse, before $\mathrm{B}$ reaches $M P$.

$$
\text { free }_{\text {temp }, A B}=\left(t_{\overline{B M P}}>t_{\overline{A M P}}+\Delta T_{A B}\right)
$$

In order to be the second to enter the critical area, the following two conditions have to be met:

1. At time $t_{\overline{B P}_{B 2}}$, when $\mathrm{B}$ reaches $P_{B 2}$, A may not have passed $P_{A 1}$ yet.

$$
\text { free }_{\text {spat }, B A}=\left(d_{\overline{A P}}\left(t_{\overline{B P}_{B 2}}\right)<d_{A}-D_{A 1}\right)
$$

2. After $\mathrm{B}$ has passed $M P$, a given time span $\Delta T_{B A}$ has to elapse, before A reaches $M P$.

$$
\text { free }_{\text {temp }, B A}=\left(t_{\overline{A M P}}>t_{\overline{B M P}}+\Delta T_{B A}\right)
$$

This means if

$$
\text { free }=\left(\text { free }_{\mathrm{spat}, A B} \wedge \text { free }_{\mathrm{temp}, A B}\right) \vee\left(\text { free }_{\mathrm{spat}, B A} \wedge \text { free }_{\mathrm{temp}, B A}\right)
$$

is true, it is assured that neither $A$ is between $P_{A 1}$ and $P_{A 2}$ as long as $B$ is between $P_{B 1}$ and $P_{B 2}$ nor the time gaps in $M P$ are shorter than permitted.

The extension from a single vehicle $B$ to $n$ vehicles $B_{i}$ is straightforward As long as one vehicle fails the verification, $A$ is not allowed to enter the critical zone:

$$
\text { free }_{\text {tot }}=\text { free }_{1} \wedge \text { free }_{2} \wedge \cdots \wedge \text { free }_{n}
$$

\subsection{Integration into the state machine}

The planner of Sec. 8 always deploys the Moving Traffic Check (MTC) when AnnieWAY might come into conflict with other traffic participants demanding the same traffic space (conflict spaces). Contingent upon the result obtained from the MTC and the particular situation (conflict situations), state transitions are triggered and the resulting state generates the desired path and approves the free section for the longitudinal control.

In order to prevent frequent switching back and forth between states due to measurement noise and control inaccuracy, hysteresis in the MTC is introduced by slightly reducing the requirements once the autonomous vehicle set itself in motion. 


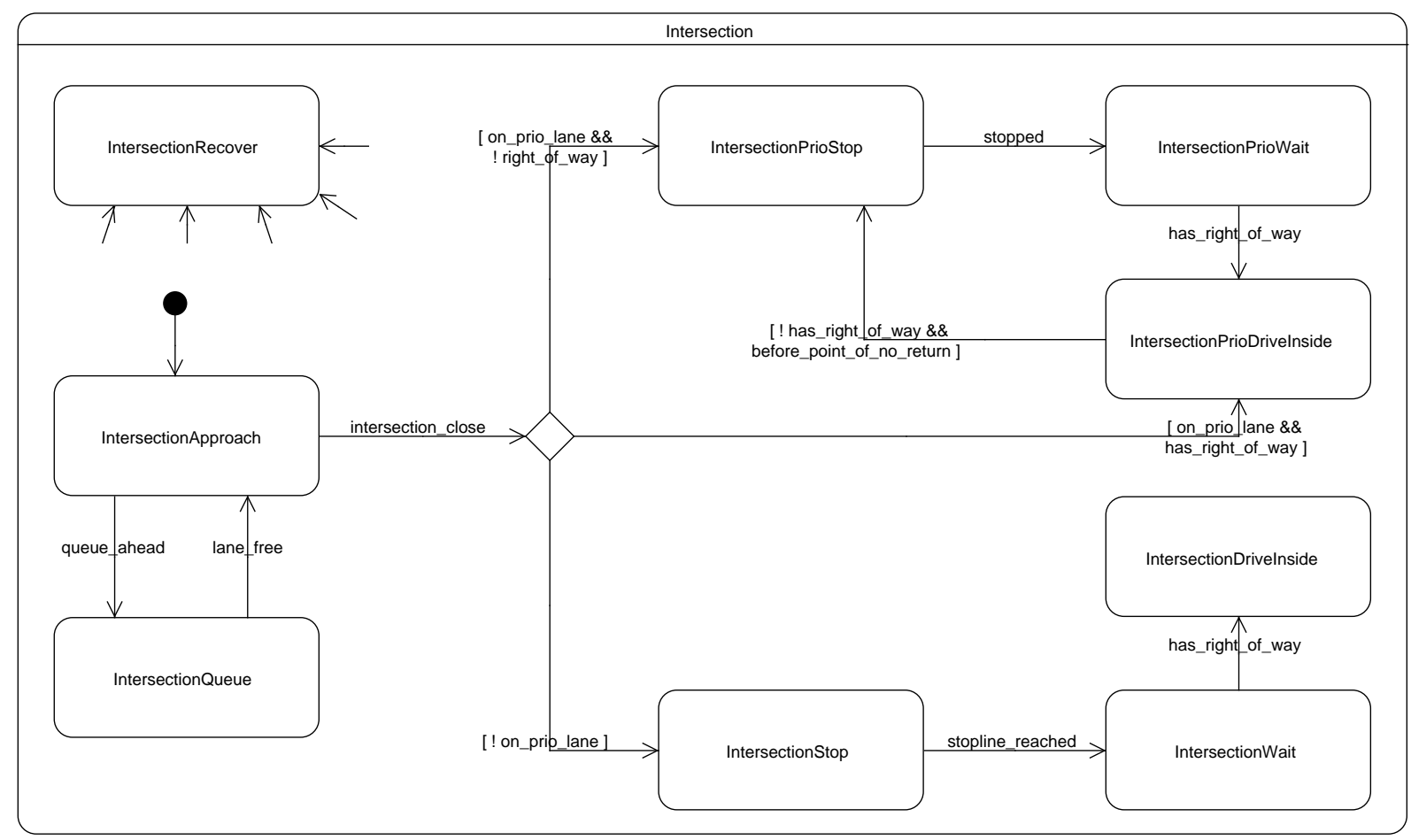

Figure 14: UML diagram of substate Intersection

Since the actual behavior of the other traffic participants can be roughly predicted at best, additional safety layers are introduced that prevent imminent collisions (see Sec. 8), in ticklish situations with emergency braking.

The conflict situations that arise from the competition, are limited to

- intersections,

- passing other cars,

- and changing lanes.

Due to the general formulation of the MTC, the different traffic situations can be accounted for with a corresponding parameter set.

For expository purposes the integration of the MTC in the intersection scenario will be described. Fig. 14 shows the corresponding block diagram in UML notation. When the vehicle approaches the intersection, the hierarchical state machine changes into the substate Intersection with the entry state IntersectionApproach. This state is active until the vehicle enters the intersection unless another traffic participant is perceived on the same lane between AnnieWAY and the intersection. In this case ItersectionQueue is activated until the other vehicle has passed the intersection and the lane is free.

In IntersectionApproach, as soon as AnnieWAY gets close to the intersection, the state transition splits up into 
(a) IntersectionStop if AnnieWAY is on a stop road,

(b) IntersectionPrioDriveInside if AnnieWAY is on a priority road and no other vehicle has the right of way,

(c) or IntersectionPrioStop if AnnieWAY is situated on a priority road, but needs to yield the right of way for a priority vehicles, e.g. approaching traffic, bevor it may turn left.

In case (a) AnnieWAY stops at the stop line and changes into the state Intersection Wait. In this state all vehicles are registered that are already waiting on another stop line which have the right of way according to the driving rules (4-Way-Stop). As soon as these vehicles have passed the intersection and the MTC turns out positive for all visible priority vehicles, the state machine changes to IntersectionDriveInside and AnnieWAY merges into the moving traffic according to the safety parameters.

In case (b) AnnieWAY drives into the intersection without stopping. If a priority vehicle is perceived shortly after driving inside the intersection (point of no return has not been passed yet) and the MTC turns out negative, the state machine switches to IntersectionPrioStop which is equivalent to (c).

In case (c) in IntersectionPrioStop AnnieWAY stops before crossing the opposing lane, waits until the MTC confirms that no danger comes from priority vehicles anymore, and turns left. 


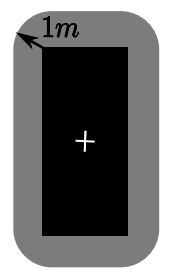

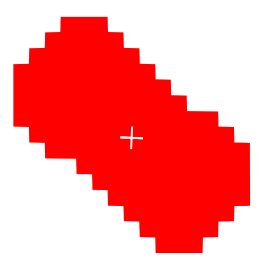

(a)

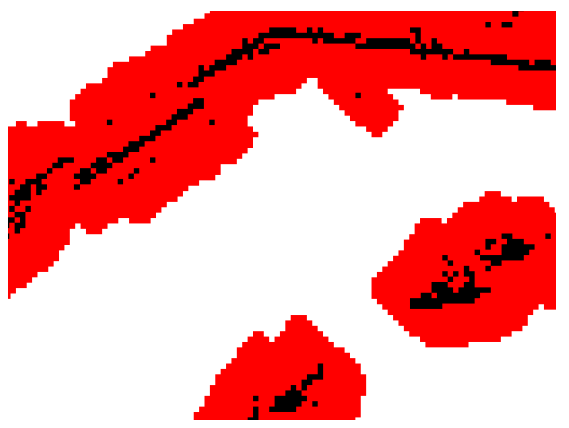

(b)

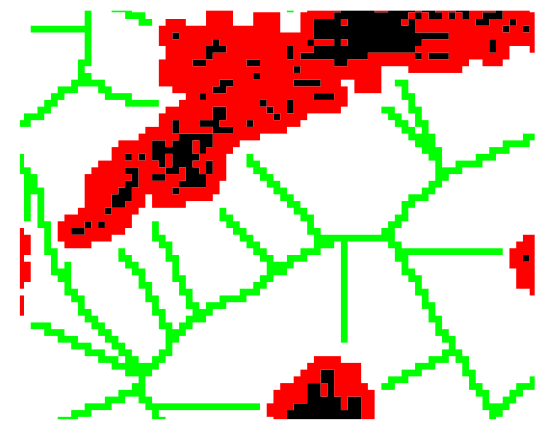

(c)

Figure 15: Configuration space obstacles. (a): A $1 \mathrm{~m}$ safety distance is added to the shape of the vehicle. Subsequent rotation and rasterization yields a convolution kernel for configuration space obstacle generation. (b): Result of convolving obstacle map with kernel from (a). If the robot has the same orientation as the kernel and is placed in the red area, it must intersect with an obstacle. (c): Voronoj lines are generated as a set of 8-connected pixels.

\section{Navigation in unstructured environment and parking}

As has been described in Sec. 8, paths can be generated in a straight forward way by sampling from the geometric road network graph where sufficient road geometry information is available. However, UC regulations require for navigating in unstructured environments (zones) that are only described by a boundary polygon. In UC, Zones are used to outline parking lots and off-road areas. In this kind of area, a graph for path planning is not available. AnnieWAY's navigation system comprises a path planning algorithm that transcends the requirement for precise road geometry definition. It has also proven useful to plan narrow turns and as a general recovery mechanism when the vehicle gets off track, the road is blocked or a sensible localization within the given road network is impossible.

\subsection{Configuration space obstacles}

We restrict search to the collision free subset of configuration space (the vehicles free space) by calculating configuration space obstacles from an obstacle map obtained from a $360^{\circ}$-laser range scanner (cf. Sec. 4.1). The discrete nature of this obstacle map motivated dealing with configuration space obstacles in a discrete way as well (Kavraki, 1995), as opposed to more traditional approaches that require obstacle input in the form of polygonal data (Schwartz and Sharir, 1983; Šwestka and Overmars, 1997). Figs. 15(a) and 15(b) illustrate how the robots free space can be generated for a discrete set of orientations. By precomputing the free space in discretized form, a collision check for a certain configuration can be performed quickly in $\mathcal{O}(1)$ by a simple table lookup. 


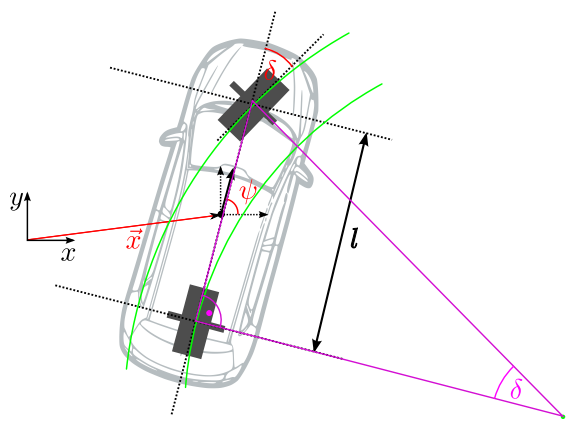

(a)

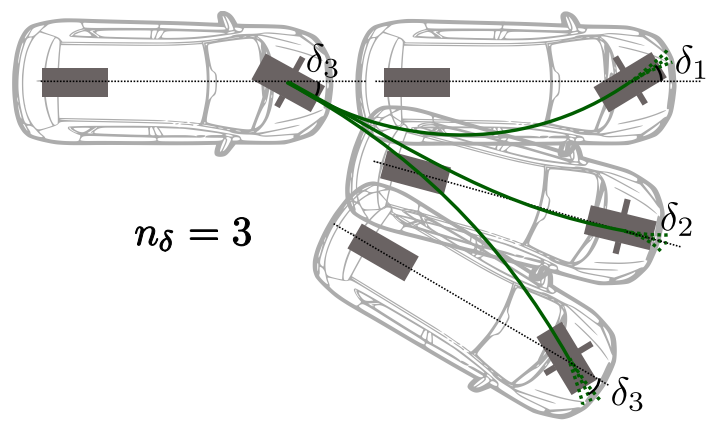

(b)

Figure 16: (a) Kinematic one track model underlying both search graph and closed loop control. (b) Search graph. Successors are generated for $n_{\delta}$ discrete steering angles.

\subsection{Search graph and $\mathrm{A}^{*}$}

We define an implicit search graph in which all paths are feasible. It is directly derived from a kinematic model of the car and not only guarantees feasibility of the generated path, but also allows for straight forward design of a combined feed forward/feed backward controller (see Sec. 11).

A node of the search graph can be completely described by a tuple $(\mathbf{x}, \psi, \delta)$, with $\mathbf{x}, \psi$ and $\delta$ denoting position, orientation and steering angle (i.e. the deflection of the front wheels) of an instance of a kinematic one track model (see Fig. 16(a)). Steering angle $\delta$ is from a set of $n_{\delta}$ discrete steering angles that are distributed equidistantly over the range of feasible steering: $D=\left\{\delta_{1} \ldots \delta_{n_{\delta}}\right\}$. To generate successors of a node, the kinematic model equations are solved for initial values taken from the node, a fixed arc length $s$ and a constant steering rate $\dot{\delta}=\frac{\delta_{p}-\delta_{i}}{s}$, spanning clothoid like arcs between the nodes. It is equivalent of driving the car model over a distance $s$ at constant speed while uniformly turning the front wheels from $\delta_{p}$ to $\delta_{i}$. For the set of nodes $\left\{\left(\mathbf{0}, 0, \delta_{i}\right), \delta_{i} \in D\right\}$, this results in $n_{\delta}^{2}$ successors, and another $n_{\delta}^{2}$ if backward motion is allowed. Successors of other nodes can be generated quickly from this precomputed set by subsequent rotation and translation (cf. Fig. 16(b)).

The search graph is expanded in this way by an $A^{*}$ search algorithm. A* search is a well known concept in the domain of robotic path planning (Hwang and Ahuja, 1992), that allows for accelerating exploration of the search space by defining a heuristic cost function that gives expected cost-to-go for each node of the search graph. If the cost function underestimates the actual distance to the goal, $\mathrm{A}^{*}$ is guaranteed to find the least-cost path. If the error of the cost function is big, $\mathrm{A}^{*}$ quickly degenerates to an exponential time algorithm. This is common when a metric cost function is used and search gets stuck in a dead end configuration. We avoid this problem by designing an obstacle sensitive cost function that accounts for the topology of the free space. 


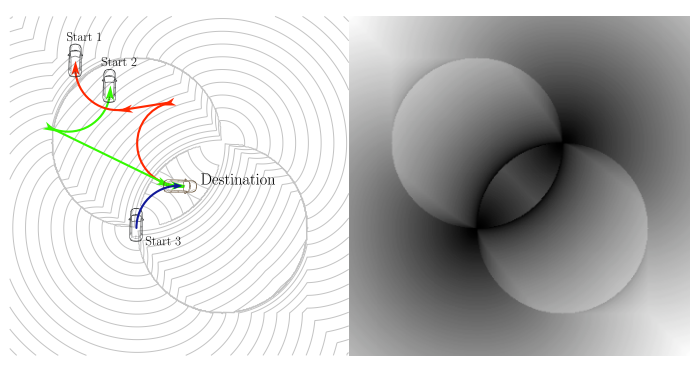

(a)
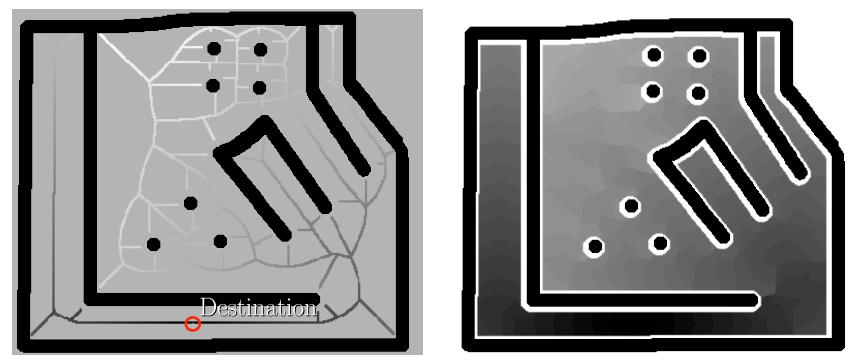

(b)

Figure 17: Cost functions. (a): RTR-metric for three different starting positions. Left hand side shows the minimum RTR-paths, right image the value of the RTR metric, densely evaluated on $\mathbb{R}^{2}$ (bright: high value, dark: low value). (b): Voronoj based cost function. Left: Voronoj graph labeled with distance by Dijkstras algorithm. Right: Voronoj based cost function evaluated densely on $\mathbb{R}^{2}$ by matching to the Voronoj-graph.

\subsection{Cost function}

To guide the search process, we combined two different cost functions. The first one accounts for kinematic constraints of the vehicle, while the second one is derived from the Voronoj graph of the vehicle's free space and thus incorporates knowledge of shape and position of the obstacles.

\subsubsection{Local cost function}

As a local cost function, the so called RTR metric is used. RTR paths connect two configurations by two circular arcs of minimum turning radius and a straight segment tangent to both. It can be shown easily (cf. (Šwestka and Overmars, 1997)), that for every pair of configurations a finite number of such paths can be constructed. The RTR metric is the arclength of the shortest such path. RTR paths do neither have continous curvature nor are they optimal (the optimal - in terms of arclength - solution to the local navigation problem are the so called Reeds and Shepp paths, cf. (Reeds and Shepp, 1991)), but are preferred by us due to their computational simplicity. Fig. 17(a) illustrates RTR metric.

\subsubsection{Voronoj based cost function}

We construct a powerful, obstacle sensitive cost function based on the Voronoj graph of the free space of the vehicle. Actually, a superset of the free space is used that is invariant to the vehicles orientation. It is generated by generating configuration space obstacles for a disk shaped structure that is the intersection of all structuring elements from Fig. 15(a).

Our algorithm to calculate Voronoj lines from a binarized obstacle map is similar to (Li and Vossepoel, 1998), however, instead of using the vector distance map, we use the approximate chamfer metric to be able to label Voronoj lines using only two passes over the obstacle map. The method is derived from an algorithm (Borgefors, 1986; Li and Vossepoel, 1998) for calculating the euclidean distance transform. It gives the Voronoj lines as a set of 8- 
connected pixels.

After matching the target position to the closest point on the Voronoj graph, Dijkstras algorithm is used to calculate the shortest path distance to the target position for every

point on the graph. Cost for a positions not on the graph is derived by matching to the closest point on the graph and incorporating the matching distance in a way that leads to a gradient of the cost function that is slightly sloped towards the Voronoj lines. Fig. 17(b) shows an example.

Using this heuristic function is appealing for several reasons. Since the Voronoj lines comprise the complete topology of the free space, search cannot get stuck in a dead end configuration, as is common with conventional, metric heuristics that do not incorporate knowledge of free space topology and therefore grossly underestimate the cost in such a case. Additionally, the Voronoj lines have - as the centers of maximum inscribing circles - the property of being at the farthest distances possible from any obstacle. This is conveyed to the planned paths, giving reserves to account for control- and measurement errors.

\subsubsection{Combination of cost functions}

We combine the two cost functions into one by the maximum operator. This procedure can be justified from the admissibility principle for heuristics in the context of $\mathrm{A}^{*}$ search. A heuristic is called admissible, if it consistently underestimates the cost to the target node. Consequently, combining two heuristics via the maximum operator still gives an admissible heuristic. Result of comparing both cost coincides with the practical experience that in the vicinity of the target position, cost is dominated by the necessity to maneuver in order to reach the destination in right orientation, while cost at long distances often is caused by the necessity to avoid obstacles. Fig. 10.3.3 shows some results of $\mathrm{A}^{*}$ search using the search graph from Sec. 10.2 and the combined cost function. 


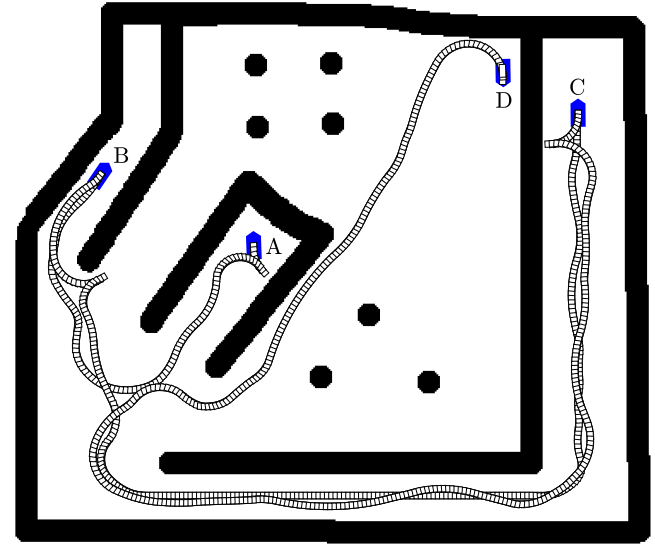

(a)

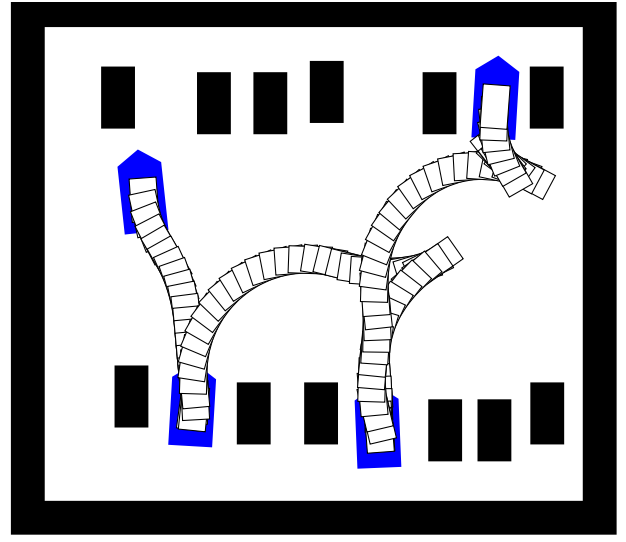

(b)

Figure 18: Some results of path planning on simulated map data. (a): Navigating long distances in a maze like environment. Planning was from A to B, B to C and C to D subsequently. (b): Some difficult parking maneuvers performed subsequently. Robot started on the right. 


\section{Vehicle Control}

The last step of the processing chain is the vehicle control which can be separated into lateral and longitudinal control. Since the distances to dynamic objects are fairly big in the Urban Challenge 2007 competition, for high-level decision making the problem of trajectory planning (coordinates of the desired vehicle position as a function of time) can be reduced to a combination of path planning (path geometries with no time dependencies) and determining the free section of the path rather than an exact desired position. The longitudinal strategy is thereby assigned to a lower level, which evaluates the free section of the path and induces the vehicle to go faster or slower. The information transfer of the interface is undertaken by so-called curve points, a discrete representation of the path geometry.

As the emphasis of the competition is on low to medium velocities, the non-holonomic single track model holds and an orbital tracking controller (e.g. (Müller, 2007)) is chosen for the lateral dynamics in Sec. 11.1. This offers the advantage of a velocity independent transient lateral behavior for the closed loop system. Suppose the vehicle had an offset from the planned path of a couple centimeters caused by sensor drift of the navigation system, the lateral controller would reduce the error over a certain traveled distance rather than over time and avoids unpredictable overshoots of the front end which might lead to collisions.

From the longitudinal controller's point of view, the vehicle drives on rails, as the lateral controller minimizes the lateral offset. Thus, the longitudinal control strategy faces solely the task of following moving objects, stopping at certain points, maintaining the maximum speed, and changing direction along the given path. For this purpose different controllers are designed in Sec. 11.2 that are included in an override control strategy ensuring bumpless transfers between them. The output of every longitudinal controller is the vehicle's acceleration $a$. This acceleration will be converted to the manipulated variables accelerator pedal value $\phi_{\text {gas }}$ and brake pressure $p_{\text {brake }}$ in a cascaded acceleration controller exceeding the scope of this contribution.

\subsection{Orbital tracking controller}

The dynamics of a non-holonomic vehicle (Fig. 19) in local coordinates $s_{c}, d$, and $\Delta \psi$ are given by

$$
\frac{\mathrm{d}}{\mathrm{d} t}\left[\begin{array}{c}
s_{c} \\
d \\
\Delta \psi
\end{array}\right]=\left[\begin{array}{c}
\frac{\cos \Delta \psi}{1-d \kappa_{c}\left(s_{c}\right)} \\
\sin \Delta \psi \\
\frac{\tan \delta}{l}-\kappa_{c}\left(s_{c}\right) \frac{\cos \Delta \psi}{1-d \kappa_{c}\left(s_{c}\right)}
\end{array}\right] v
$$

whereas the steering wheel angel $\delta$ and the longitudinal velocity $v$ is the system's input, $d$ is the lateral offset to the path, $\Delta \psi$ is the angle between the vehicle and the tangent to the path, and $l$ the distance between the rear and the front axle. The singularity at $1-d \kappa_{c}\left(s_{c}\right)=0$ is no restriction in practice since $d \ll \frac{1}{\kappa_{c}\left(s_{c}\right)}$.

Since orbital tracking control does not have any time dependencies, (18) can be rewritten with the arc length $s_{c}$ as the new time parametrization. 


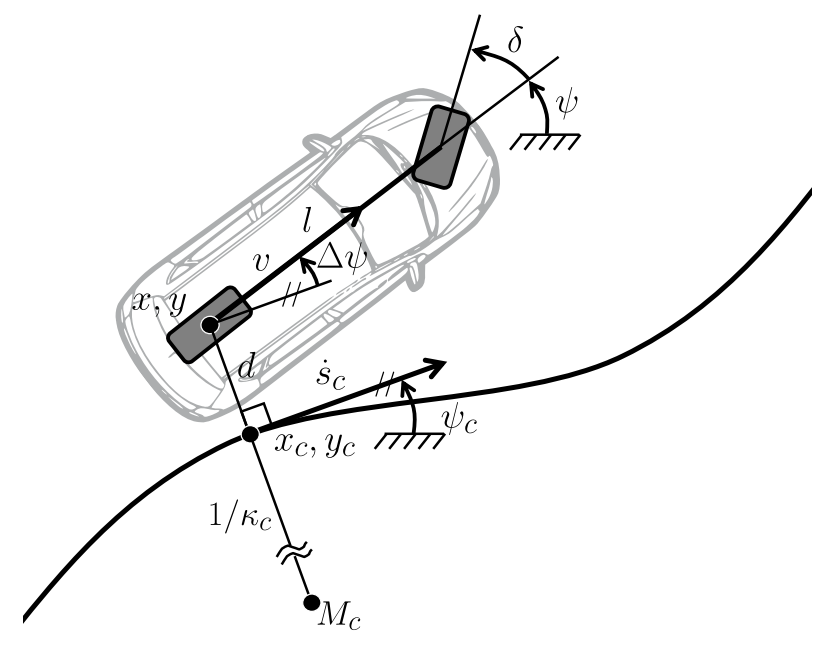

Figure 19: Non-holonomic one track model

With $\frac{\mathrm{d}}{\mathrm{d} t}()=\frac{\mathrm{d}}{\mathrm{d} s_{c}}() \cdot \frac{\mathrm{d} s_{c}}{\mathrm{~d} t}$ it becomes

$$
\frac{\mathrm{d}}{\mathrm{d} s_{c}}\left[\begin{array}{c}
s_{c} \\
d \\
\Delta \psi
\end{array}\right]=\left[\begin{array}{c}
1 \\
\sin \Delta \psi \cdot \frac{1-d \kappa_{c}\left(s_{c}\right)}{\cos \Delta \psi} \\
\frac{\tan \delta}{l} \cdot \frac{1-d \kappa_{c}\left(s_{c}\right)}{\cos \Delta \psi}-\kappa_{c}\left(s_{c}\right)
\end{array}\right] .
$$

For small deviations $d$ and $\Delta \psi$ from the desired curve and $\frac{\mathrm{d}}{\mathrm{d} s_{c}}()=()^{\prime}$, a partial linearization leads to

$$
\left[\begin{array}{c}
d \\
\Delta \psi
\end{array}\right]^{\prime}=\left[\begin{array}{ll}
0 & 1 \\
0 & 0
\end{array}\right]\left[\begin{array}{c}
d \\
\Delta \psi
\end{array}\right]+\left[\begin{array}{c}
0 \\
-1
\end{array}\right] \kappa_{c}+\left[\begin{array}{c}
0 \\
\frac{1}{l}
\end{array}\right] \tan \delta
$$

The linearizing control law

$$
\begin{aligned}
\delta & =\arctan \left(-l k_{0} d-l k_{1} \Delta \psi+l \kappa_{c}\right) \\
& =\arctan \left(-k_{1}^{\star} d-k_{2}^{\star} \Delta \psi+l \kappa_{c}\right)
\end{aligned}
$$

with $k_{0}, k_{1}>0$ yields the stable linear error dynamics

$$
\frac{\mathrm{d}}{\mathrm{d} s_{c}}\left[\begin{array}{c}
d \\
\Delta \psi
\end{array}\right]=\left[\begin{array}{cc}
0 & 1 \\
-k_{0} & -k_{1}
\end{array}\right]\left[\begin{array}{c}
d \\
\Delta \psi
\end{array}\right]
$$

with respect to $s_{c}$ with the characteristic polynomial $\lambda^{2}+k_{1} \lambda+k_{0}=0$. As long as $\dot{s}_{c}>0$, the system is also stable with respect to time. For backward driving the signs of $k_{0}$ and $k_{1}$ have to be adjusted to the applied sign convention and yields exactly the same error dynamics as for forward driving.

Fig. 20 shows the transient behavior to different initial errors $\Delta \psi$ and $d$ for forward (blue) and backward driving (red) simulated with MATLAB/SIMULINK. As parameters for the simulation the Passat's axis distance $l=2.72$, a maximum steering angle of $\delta_{\max }=30^{\circ}$, the controller parameters $k_{0}=0.25 l$ and $k_{1}=1.25 l$ and equidistant curve point with $\Delta=2 \mathrm{~m}$ were chosen. Obviously neither the input saturation $\delta_{\max }$ nor the discrete representation of the curve cause any significant problems. 


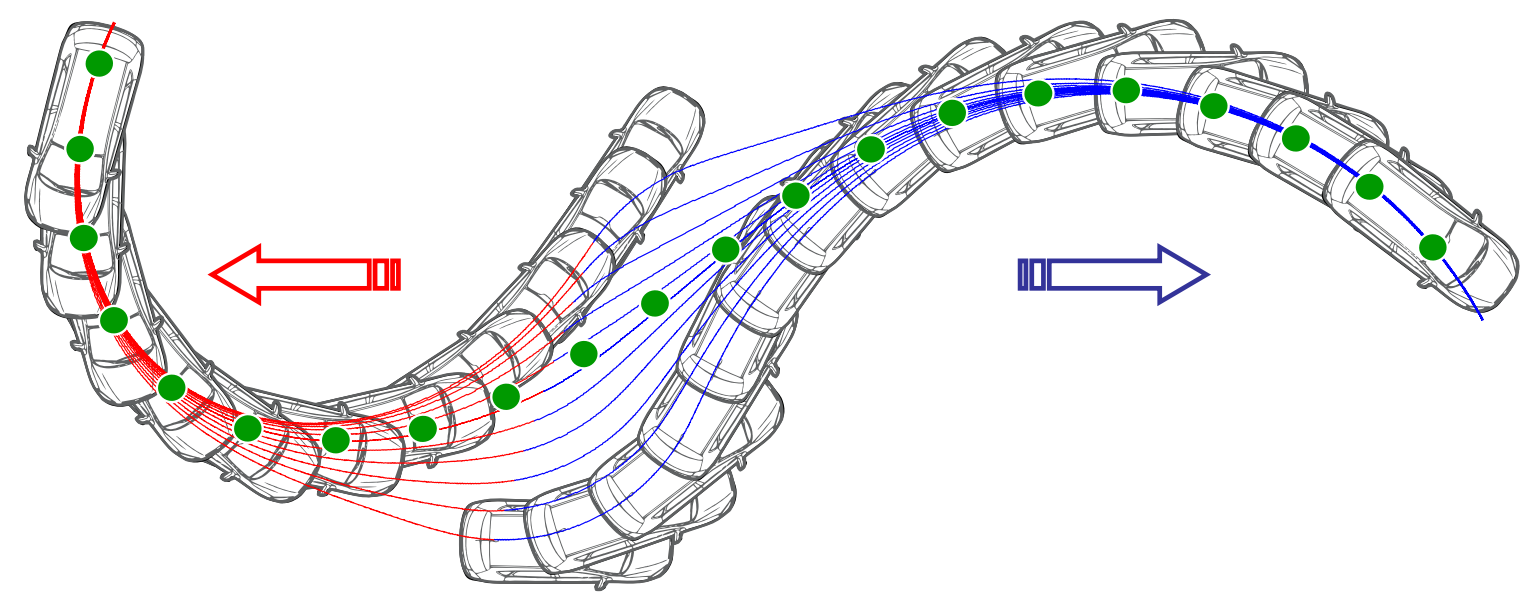

Figure 20: Trajectories for different initial positions.

\subsection{Longitudinal controller system}

\subsubsection{Following controller}

Since the acceleration of a leading vehicle is hard to determine, it is assumed that the vehicle keeps its velocity $v_{B}$ constant. Choosing the distance $d_{f}$ and its time derivative $\dot{d}_{f}$ as the state variables and AnnieWAY's acceleration $a_{f}=\dot{v}$ as the input, the system's dynamics are given by

$$
\frac{\mathrm{d}}{\mathrm{d} t}\left[\begin{array}{c}
d_{f} \\
\dot{d}_{f}
\end{array}\right]=\left[\begin{array}{ll}
0 & 1 \\
0 & 0
\end{array}\right]\left[\begin{array}{c}
d_{f} \\
\dot{d}_{f}
\end{array}\right]+\left[\begin{array}{c}
0 \\
-1
\end{array}\right] a_{f}
$$

As DARPA requires the vehicle to maintain a minimum forward vehicle separation of one vehicle length minimum and one length for every additional $10 \mathrm{mph}$, the desired distance $d_{f, d}$ can be calculated by

$$
d_{f, d}=d_{f, 0}+\tau v
$$

with the according parameters $d_{f, 0}$ and $\tau$. Considering the acceleration $\dot{v}_{B}$ of the leading vehicle an unmeasurable disturbance, the linear set-point control law

$$
\begin{aligned}
a_{f} & =c_{0}\left(d_{f}-d_{f, d}\right)+c_{1} \dot{d}_{f} \\
& =c_{0}\left(d_{f}-d_{f, d}\right)+c_{1}\left(v_{B}-v\right)
\end{aligned}
$$

and $v=v_{B}-\dot{d}_{f}$ yields the total system

$$
\frac{\mathrm{d}}{\mathrm{d} t}\left[\begin{array}{c}
d_{f} \\
\dot{d}_{f}
\end{array}\right]=\left[\begin{array}{cc}
0 & 1 \\
-c_{0} & -c_{0} \tau-c_{1}
\end{array}\right]\left[\begin{array}{c}
d_{f} \\
\dot{d}_{f}
\end{array}\right]+\left[\begin{array}{c}
0 \\
c_{0}\left(d_{f, 0}+\tau v_{B}\right)
\end{array}\right] .
$$

The characteristic polynomial $\lambda^{2}+\left(c_{0} \tau+c_{1}\right) \lambda+c_{0}=0$ can directly be read off from (28). A double Eigenvalue $\lambda_{1 / 2}=-1$ leads to a pleasant an yet save following behavior. 


\subsubsection{Stopping controller}

The following controller of the previous section leads to a behavior, which can best be described as flowing with the traffic. By contrast, the stopping controller should come to a controlled stop at a certain point as fast as possible without exceeding any comfort criteria. The control law

$$
a_{s}=-\frac{v^{2}}{2\left(d_{f}-d_{\Delta}\right)}
$$

leads to a constant deceleration until the vehicle is $d_{\Delta}$ away from the stop point. To prevent the controller from decelerating too soon and switching on and off, a hysteresis with the thresholds $a_{s, \max }$ and $a_{s, \min }$, as shown in Fig. 21, is introduced. The singularity at $d_{f}=d_{\Delta}$ is avoided by a PD position controller that takes over via a min-operator and ensures a smooth and save stop at the end.

\subsubsection{Velocity controller}

As $\dot{v}=a$, the simple proportional velocity control law

$$
a_{v}=-c_{v}\left(v-v_{d}\right)
$$

stabilizes AnnieWAY's velocity $v$ to the desired velocity $v_{d}$ with a $P T_{1}$ behavior.

\subsubsection{Override control strategy}

All three previously introduced controllers are combined by an override control strategy depicted in Fig. 21. The bumpless transfer between velocity control and following/stopping control is assure by the max operator. Additional saturation, realized by $a_{\max }$ and $a_{\min }$, prevent the vehicle from inappropriately high acceleration or deceleration without reducing safety.

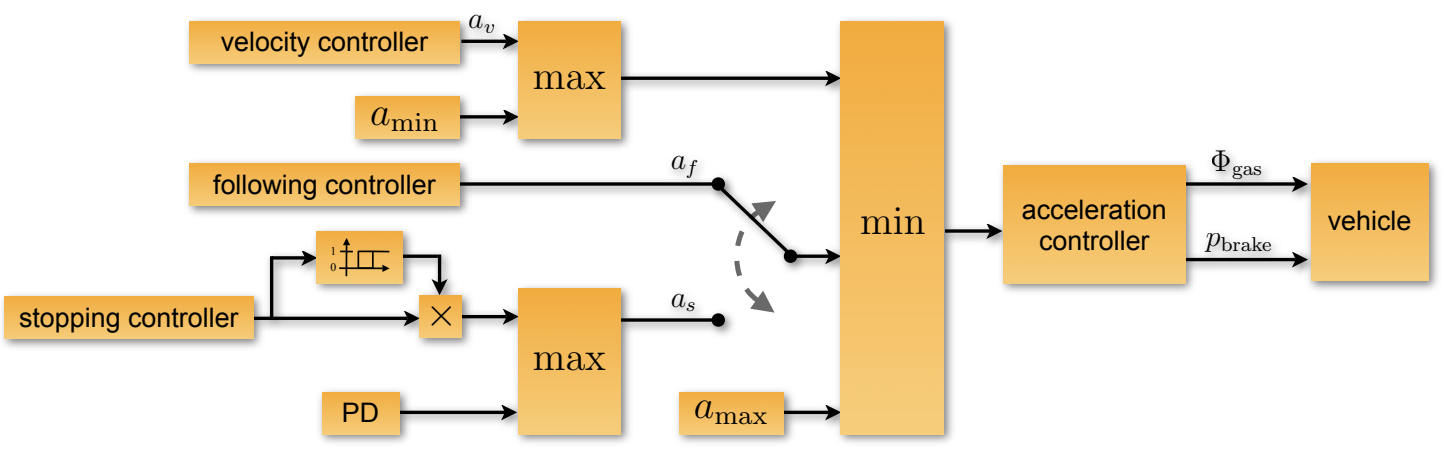

Figure 21: Longitudinal override control strategy. 


\section{Results}

Originally 89 teams have entered the competition, 11 of which were sponsored by the organizer. After several stages, 36 of those teams were selected for the semi-final. There, AnnieWAY has accomplished safe conduction of a variety of maneuvers including

- regular driving on lanes

- turning at intersections with oncoming traffic

- lane change maneuvers

- vehicle following and passing

- following order of precedence at 4-way stops

- merging into moving traffic

Although the final event was originally planned to challenge 20 teams, only 11 finalists were selected by the organizers due to safety issues. AnnieWAY has entered the final and was able to conduct a variety of driving maneuvers. It drove collision-free, but stopped due to a software exception in one of the modules.

Fig. 22 depicts three examples of the vehicles actual course taken from a log-file and superimposed on an aerial image. The rightmost figure shows the stopping position in the finals.
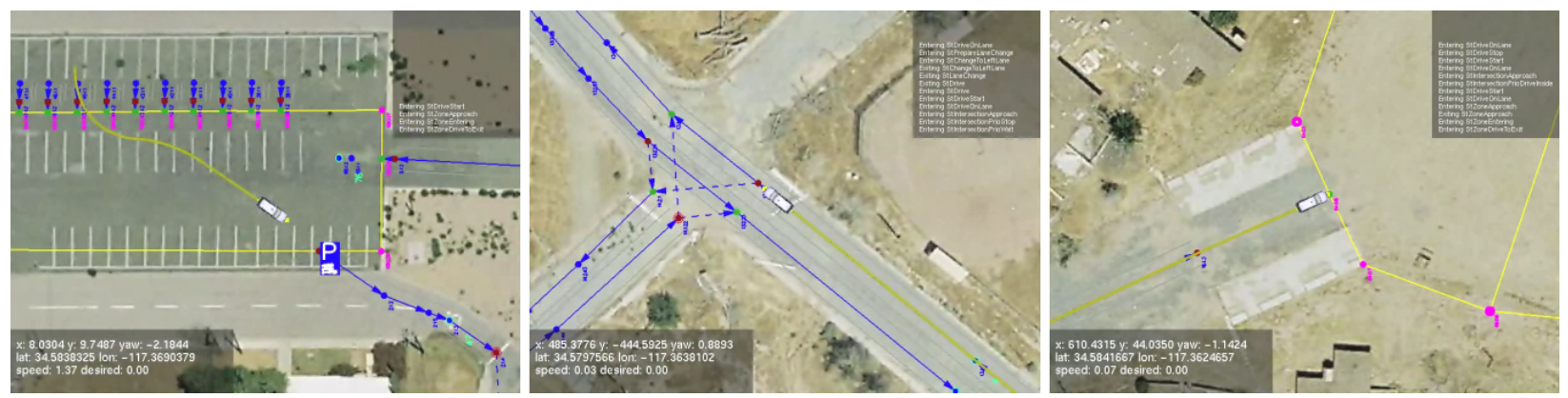

Figure 22: Three steps of AnnieWAYs course driven autonomously in the finals.

The following section points out some results of the navigation module. Fig. 23(a) illustrates one test driven in a parking area close to our test ground. Unlike the required navigation task in the Urban Challenge, the chosen setup features many surrounding obstacles such as other cars and curbs.

Search time remains below 2 seconds in all practical situations. Though the environment is assumed to be static, this is fast enough to cope with slow changes in the environment by continuous replanning. Additionally, to avoid collision with fast moving objects, a lower level process continuously determines the free section of the planned path and, if necessary, invokes a new search. The lateral controller follows the generated paths precisely enough to implement all of the intended maneuvers. 

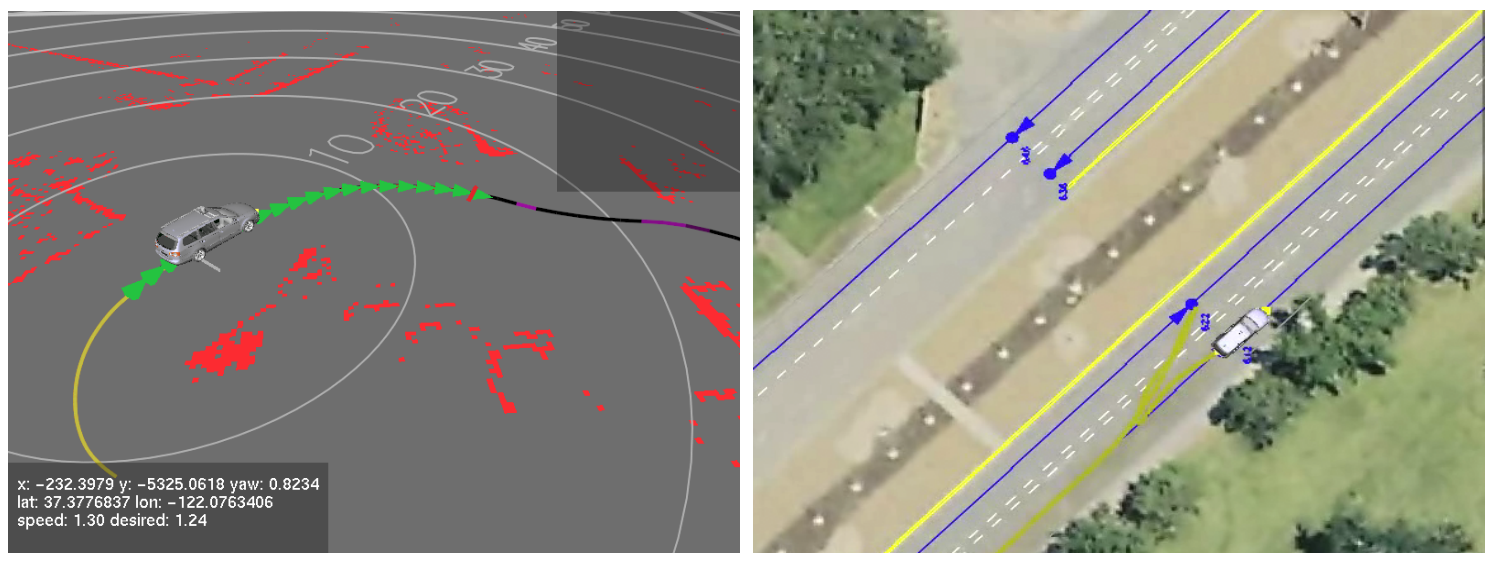

Figure 23: (a) Path planning in heavily occupied zone with mapper input (red) and sampled waypoints as output to the controller (green). (b) Recovery maneuver during final event, driven path is marked olive.

Besides path planning in parking areas, the zone-navigation module was used as recovery option in case of continuous blocking of lanes or intersections. A falsely detected obstacle on the left lane forced activation of the navigation module during the final event. The vehicle was successfully brought back on track after a backup maneuver as can be seen in Fig. 23(b). 


\section{Conclusions}

The autonomous vehicle AnnieWAY is capable of driving through urban scenarios and has successfully entered the finals of the DARPA Urban Challenge 2007 competition. In contrast to earlier competitions, the Urban Challenge required to conduct missions in 'urban' traffic, i.e. in the presence of other autonomous and human-operated vehicles. The major challenge imposed was collision-free and rule-compliant driving in traffic. AnnieWAY is based on a simple and robust hardware architecture. In particular, we rely on a single computer system for all tasks but low level control. Environment perception is mainly conducted by a roof-mounted laser scanner that measures range and reflectivity for each pixel. While the former is used to provide 3D scene geometry, the latter allows robust lane marker detection. Mission and maneuver selection is conducted via a concurrent hierarchical state machine that specifically ascertains behavior in accordance with California traffic laws. More than 100 hours of urban driving without human intervention in complex urban settings with multiple cars, correct precedence order decision at intersections and - last not least - the entry in the finals underline the performance of the overall system. 


\section{Acknowledgments}

The authors gratefully acknowledge the fruitful collaboration of their partners from Universität Karlsruhe, Technische Universität München and Universität der Bundeswehr München. Special thanks are directed to Annie Lien for her instant willingness and dedication as our official team leader. This work had not been possible without the ongoing research of the Transregional Collaborative Research Centre 28 'Cognitive Automobiles'. Both projects cross-fertilized each other and revealed significant synergy. The authors gratefully acknowledge support of the TCRC by the Deutsche Forschungsgemeinschaft (German Research Foundation).

\section{References}

Bertozzi, M., Broggi, A., and Fascioli, A. (2000). Vision-based intelligent vehicles: State of the art and perspectives. J. of Robotics and Autonomous Systems, 32:1-16.

Biber, P. and Strasser, W. (2006). nscan-matching: Simultaneous matching of multiple scans and application to slam. In IEEE International Conference on Robotics and Automation.

Borgefors, G. (1986). Distance transformations in digital images. Computer Vision, Graphics, and Image Processing, 34(3):344-371.

Bosse, M., Newman, P. M., Leonard, J. J., Soika, M., Feiten, W., and Teller, S. J. (2003). An atlas framework for scalable mapping. In Proceedings of the 2003 IEEE International Conference on Robotics and Automation, pages 1899-1906, Taipei, Taiwan.

Bracewell, R. N. (1990). Numerical transforms. Science, 248(4956):697-704.

DARPA (2005). Grand Challenge '05. http://www.darpa.mil/ grandchallenge/overview.html.

Dickmanns, E. D., Behringer, R., Dickmanns, D., Hildebrandt, T., Maurer, M., Thomanek, F., and Schielen, J. (1994). The seeing passenger car "VaMoRs-P". In Proc. Symp. On Intelligent Vehicles, pages 68-73, Paris.

Franke, U., Gavrila, D., Gern, A., Goerzig, S., Jansen, R., Paetzold, F., and Wöhler, C. (2001). From door to door - Principles and applications of computer vision for driver assistant systems. In Vlacic, L., Harashima, F., and Parent, M., editors, Intelligent Vehicle Technologies: Theory and Applications, chapter 6, pages 131-188. Butterworth Heinemann, Oxford.

Goebl, M. and Färber, G. (2007a). Eine realzeitfähige Softwarearchitektur für kognitive Automobile. In Berns, K. and Luksch, T., editors, Autonome Mobile Systeme 2007, Informatik Aktuell, pages 198-204. "Springer-Verlag".

Goebl, M. and Färber, G. (2007b). A real-time-capable hard- and software architecture for joint image and knowledge processing in cognitive automobiles. In Proc. IEEE Intelligent Vehicles Symposium, pages 734-739, Istanbul, Turkey.

Hummel, B., Kammel, S., Dang, T., Duchow, C., and Stiller, C. (2006). Vision-based pathplanning in unstructured environments. In IEEE Intelligent Vehicles Symposium, Tokyo, Japan. 
Hwang, Y. K. and Ahuja, N. (1992). Gross motion planning - a survey. ACM Comput. Surv., 24(3):219-291.

Kalman, R. (1960). A new approach to linear filtering and prediction problems. Journal of Basic Engineering, 82(1):35-45.

Kavraki, L. (1995). Computation of configuration-space obstacles using the fast fourier transform. IEEE Transactions on Robotics and Automation, 11(3):408-413.

Li, H. and Vossepoel, A. M. (1998). Generation of the euclidean skeleton from the vector distance map by a bisector decision rule. In CVPR '98: Proceedings of the IEEE Computer Society Conference on Computer Vision and Pattern Recognition, page 66, Washington, DC, USA. IEEE Computer Society.

Müller, B.; Deutscher, J. (2007). Orbital tracking control for car parking via control of the clock. Methoden und Anwendungen der Regelungstechnik, Erlangen-Münchener Workshops 2005 und 2006, Shaker Verlag.

Nagel, H.-H., Enkelmann, W., and Struck, G. (1995). FhG-Co-Driver: From map-guided automatic driving by machine vision to a cooperative driver support. Mathematical and Computer Modelling, 22:185-212.

Özgüner, Ü., Stiller, C., and Redmill, K. (2007). Systems for safety and autonomous behavior in cars: The DARPA Grand Challenge experience. IEEE Proceedings, 95(2):1-16.

Reeds, J. and Shepp, R. (1991). Optimal paths for a car that goes both forward and backward. Pacific Journal of Mathematics, 145(2):144-154.

Schwartz, J. T. and Sharir, M. (1983). On the piano movers' problem, ii: General techniques for computing topological properties of real algebraic manifolds. Advances in Applied Mathematics, 4:298-351.

Thorpe, C. (1990). Vision and navigation - The Carnegie Mellon Navlab. Kluwer Academic Publishers.

Thrun, S. (2002). Robotic mapping: A survey. In Lakemeyer, G. and Nebel, B., editors, Exploring Artificial Intelligence in the New Millenium. Morgan Kaufmann.

Thrun, S. (2003). Learning occupancy grid maps with forward sensor models. Auton. Robots, $15(2): 111-127$.

Thrun, S., Montemerlo, M., Dahlkamp, H., Stavens, D., Aron, A., Diebel, J., Fong, P., Gale, J., Halpenny, M., Hoffmann, G., Lau, K., Oakley, C., Palatucci, M., Pratt, V., Stang, P., Strohband, S., Dupont, C., Jendrossek, L.-E., Koelen, C., Markey, C., Rummel, C., Niekerk, J., Jensen, E., Alessandrini, P., Bradski, G., Davies, B., Ettinger, S., Kaehler, A., Nefian, A., and Mahoney, P. (2006). Stanley: The robot that won the DARPA Grand Challenge. Journal of Field Robotics, 23(9):661-692.

v. Hundelshausen, F., Himmelsbach, M., Mueller, A., and Wuensche, H.-J. (2008). Tentaclesa biologically inspired approach for robot navigation. Journal of Field Robotics, page under submission.

Šwestka, P. and Overmars, M. (1997). Motion planning for car-like robots using a probabilistic learning approach. The International Journal of Robotics Research, 16(2):119-143. 